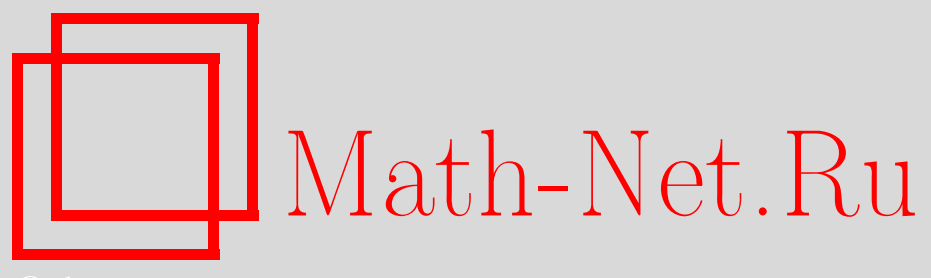

Д. В. Горбачев, В. И. Иванов, Квадратурные формулы Гаусса и Маркова по нулям собственных функций задачи Штурма-Лиувилля, точные для целых функций экспоненциального типа, Матем. сб., 2015, том 206, номер 8, 63-98

DOI: https://doi.org/10.4213/sm8413

Использование Общероссийского математического портала Math-Net.Ru подразумевает, что вы прочитали и согласны с пользовательским соглашением http://www.mathnet.ru/rus/agreement

Параметры загрузки:

IP : 54.205 .225 .156

26 апреля 2023 г., 14:28:52

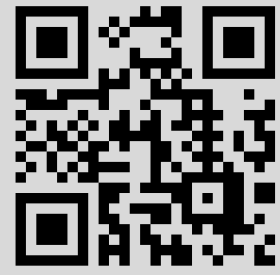




\author{
Д. В. Горбачев, В. И. Иванов
}

\title{
Квадратурные формулы Гаусса и Маркова по нулям собственных функций задачи Штурма-Лиувилля, точные для целых функций экспоненциального типа
}

Доказываются квадратурные формулы Гаусса и Маркова по нулям собственных функций задачи Штурма-Лиувилля, точные для целых функций экспоненциального типа. Они обобщают квадратурные формулы по нулям функций Бесселя, впервые построенные К. Фрапье и П. Оливье. Квадратуры Бесселя отвечают интегральному преобразованию ФурьеГанкеля. Приводятся другие примеры, связанные с интегральным преобразованием Якоби, рядом Фурье по ортогональным многочленам Якоби и общей задачей Штурма-Лиувилля с регулярным весом.

Библиография: 39 названий.

Ключевые слова: квадратурные формулы Гаусса и Маркова, целая функция экспоненциального типа, задача Штурма-Лиувилля, преобразование Якоби, функции и многочлены Якоби.

DOI: $10.4213 / \operatorname{sm} 8413$

\section{§ 1. Введение}

В математике хорошо известны квадратурные формулы Гаусса вида

$$
\int_{a}^{b} f(x) d \sigma(x)=\sum_{k=1}^{n} \gamma_{k} f\left(z_{k}\right),
$$

где интервал $(a, b) \subset \mathbb{R}$, мера $d \sigma \geqslant 0$, узлы $a<z_{1}<\cdots<z_{n}<b$, а веса $\gamma_{k}$ (числа Кристоффеля) положительны. Обычно полагают $[a, b]=[-1,1]$.

Формулам Гаусса и их вариантам посвящена обширная литература (см., например, [1]-[3]). В основном, они изучались для меры $\sigma$ с конечными моментами

$$
\int_{a}^{b} x^{j} d \sigma(x)<+\infty, \quad j \in \mathbb{Z}_{+} .
$$

В этом случае справедливость формулы (1.1) естественно требовать на классе алгебраических многочленов. Если в качестве узлов $z_{k}$ взять нули ортогонального по мере $\sigma$ многочлена $\pi_{n}(x)$ степени $n$, удовлетворяющего трехчленному рекуррентному соотношению, то формула (1.1) при соответствующих $\gamma_{k}$ будет

Работа выполнена при поддержке Российского фонда фундаментальных исследований (грант № 13-01-00045), Министерства образования и науки РФ (госзадания № 5414ГЗ, № 1.1333.2014K) и Фонда Д. Зимина “Династия". 
иметь наивысшую алгебраическую степень точности $2 n-1$. Самим К. Гауссом был рассмотрен случай $d \sigma(x)=d x$, когда $\pi_{n}$ - ортогональный многочлен Лежандра $P_{n}(x)$.

В формулах Маркова (или Гаусса-Радо) фиксируются узлы на границе промежутка интегрирования (см. [1], [3]). Например,

$$
\int_{-1}^{1} f(x) d \sigma(x)=\sum_{k=0}^{n} \gamma_{k}^{\prime} f\left(z_{k}^{\prime}\right), \quad z_{0}^{\prime}=1 .
$$

Появляется еще один вес $\gamma_{0}^{\prime}$, поэтому формула справедлива для многочленов степени, не большей $2 n$. При этом узлами $z_{k}^{\prime}$ будут нули $z_{k}^{1}$ ортогонального по мере $d \sigma_{1}=(1-x) d \sigma$ многочлена $\pi_{n}^{1}(x)$. В частности, для $d \sigma=d x$ имеем многочлен Якоби $P_{n}^{(1,0)}(x)$. Формула Маркова вытекает из формулы Гаусса

$$
\int_{-1}^{1} f d \sigma_{1}=\sum_{k=1}^{n} \gamma_{k}^{1} f\left(z_{k}^{1}\right)
$$

если ее применить к многочлену $\left(f(x)-f(1) p^{2}(x)\right) /(1-x)$ степени $2 n-1$, где $p(x)=\left(\pi_{n}^{1}(x) / \pi_{n}^{1}(1)\right)^{2}$. Весами будут

$$
\gamma_{0}^{\prime}=\int_{-1}^{1} p^{2} d \sigma, \quad \gamma_{k}^{\prime}=\frac{\gamma_{k}^{1}}{1-z_{k}^{1}}, \quad k>0 .
$$

Экстремальный характер формул Гаусса и Маркова и положительность весов в них позволяют решить многие задачи для многочленов, имеющие важные приложения в теории функций и дискретной математике (см., например, [4]-[6]). Приведем два примера.

1. Покажем, что многочлен $f$ степени $2 n-1$ с нулевым средним значением

$$
\int_{-1}^{1} f(x) d x=0, \quad f(1)>0
$$

строго положителен на промежутке $[-1, \lambda]$ при $\lambda>z_{n}$. Если это не так, то применим квадратурную формулу Гаусса:

$$
0=\int_{-1}^{1} f(x) d x=\sum_{k=1}^{n} \gamma_{k} f\left(z_{k}\right) \leqslant 0 .
$$

Таким образом, в точках $z_{k}$ многочлен $f$ имеет двойные нули, что невозможно. Отсюда следует, что $\lambda \leqslant z_{n}$. При этом единственным с точностью до положительной константы экстремальным многочленом будет $P_{n}^{2}(x)\left(x-z_{n}\right)^{-1}$.

2. Найдем $\max f(1)$, где $f$ - неотрицательный многочлен степени $2 n$ со средним значением

$$
\int_{-1}^{1} f d x=1
$$

Применим формулу Маркова:

$$
1=\int_{-1}^{1} f(x) d x=\gamma_{0}^{\prime} f(1)+\sum_{k=1}^{n} \gamma_{k}^{\prime} f\left(z_{k}^{\prime}\right) \geqslant \gamma_{0}^{\prime} f(1) .
$$


Отсюда находим, что $\max f(1) \leqslant\left(\gamma_{0}^{\prime}\right)^{-1}$. Равенство достигается на единственном с точностью до положительной константы многочлене $\left(P_{n}^{(1,0)}(x)\right)^{2}$, имеющем двойные нули в узлах $z_{k}^{1}$.

Решения приведенных экстремальных задач для многочленов важны при оценке параметров кодов и дизайнов на сфере и других компактных римановых пространствах (см. [5], [4]).

В работе изучаются квадратурные формулы Гаусса

$$
\int_{0}^{\infty} f(x) d \sigma(x)=\sum_{k=1}^{\infty} \gamma_{k} f\left(z_{k}\right), \quad 0<z_{1}<z_{2}<\cdots, \quad \gamma_{k}>0,
$$

и Маркова

$$
\int_{0}^{\infty} f(x) d \sigma(x)=\sum_{k=0}^{\infty} \gamma_{k}^{\prime} f\left(z_{k}^{\prime}\right), \quad z_{0}^{\prime}=0<z_{1}^{\prime}<z_{2}^{\prime}<\cdots, \quad \gamma_{k}^{\prime}>0,
$$

где мера $\sigma$ - неубывающая функция ограниченной вариации $(d \sigma \geqslant 0)$ с моментами

$$
\int_{0}^{\infty} x^{j} d \sigma(x)=+\infty, \quad j \in \mathbb{Z}_{+} .
$$

Рассматриваются случаи непрерывной и дискретной меры $\sigma$ :

1) $\sigma \in C^{1}\left(\mathbb{R}_{+}\right), s=\sigma^{\prime}$ - непрерывный неотрицательный вес,

$$
\int_{\mathbb{R}_{+}} f(x) d \sigma(x)=\int_{0}^{\infty} f(x) s(x) d x
$$

2) $\sigma(x)=\sum_{\zeta_{k} \leqslant x} s_{k}-$ функция скачков, $s_{k} \geqslant 0, k \in \mathbb{Z}_{+}$,

$$
\begin{gathered}
\zeta_{0}=0<\zeta_{1}<\zeta_{2}<\cdots, \quad \inf _{k \in \mathbb{Z}_{+}} \Delta \zeta_{k}>0, \quad \Delta \zeta_{k}=\zeta_{k+1}-\zeta_{k}, \\
\int_{\mathbb{R}_{+}} f(x) d \sigma(x)=\sum_{k=0}^{\infty} s_{k} f\left(\zeta_{k}\right) .
\end{gathered}
$$

При этом в непрерывном и дискретном случаях соответственно предполагается, что

$$
s(x) \asymp x^{\nu}, \quad x \rightarrow+\infty, \quad s_{k} \asymp \zeta_{k}^{\nu}, \quad k \rightarrow \infty,
$$

где показатель $\nu \geqslant 0$. Поскольку для таких мер не существует ни одного момента, точность формул (1.2) и (1.3) будет требоваться для действительных и ограниченных на $\mathbb{R}$ четных целых функций $f$ экспоненциального типа $\tau(f) \leqslant d, d>0$. Их множество обозначается через $B_{d}^{0} \subset B_{d}$, где $B_{d}-$ класс Бернштейна (см. [7; гл. IV]). Типом $\tau(f)$ целой функции $f$ называется нижняя грань чисел $a>0$, для которых справедливо неравенство $|f(z)| \leqslant C_{a} e^{a|z|}$ для Bcex $z \in \mathbb{C}$.

Пусть $L_{v}^{p}(I)$ или $L_{d \mu}^{p}(I)$ обозначают весовой класс функций $f$ с конечной нормой

$$
\|f\|_{p, v}=\left(\int_{I}|f|^{p} v d t\right)^{1 / p}
$$


или

$$
\|f\|_{p, d \mu}=\left(\int_{I}|f|^{p} d \mu\right)^{1 / p}, \quad(f, g)_{v}=\int_{I} f \bar{g} v d t, \quad(f, g)_{d \mu}=\int_{I} f \bar{g} d \mu .
$$

Из условия (1.6) следует, что если для $\delta>\nu+1$

$$
f(x)=O\left(x^{-\delta}\right), \quad x \rightarrow+\infty
$$

(или просто $f=O_{+\infty}\left(x^{-\delta}\right)$ ), то для произвольного $a>0$

$$
\int_{a}^{\infty}|f| d \sigma \lesssim \int_{a}^{\infty} x^{-\delta} d \sigma \asymp \int_{a}^{\infty} x^{-\delta+\nu} d x \asymp a^{\nu+1-\delta},
$$

где асимптотическое неравенство $\lesssim$ означает $\leqslant C$. В непрерывном случае это очевидно, а в дискретном можно воспользоваться интегральным признаком сходимости Коши-Маклорена. Поэтому $f \in L_{x^{\nu}}^{1}\left(\mathbb{R}_{+}\right) \cap L_{d \sigma}^{1}\left(\mathbb{R}_{+}\right)$. Мы часто будем пользоваться этим простым фактом.

Впервые формулы Гаусса на полуоси, точные для целых функций экспоненциального типа меньших или равных $2 \tau$, были установлены К. Фрапье и П. Оливье в [8] для случая степенного веса $s(x)=x^{2 \alpha+1}$ при $\alpha>-1$ (мы ограничимся случаем $\alpha \geqslant-1 / 2$ ). Узлами в этом случае (при $\tau=1$ ) являются положительные нули $0<q_{\alpha, 1}<q_{\alpha, 2}<\cdots$ функции Бесселя $J_{\alpha}$ (см. [9; гл. 7]). Положим $q_{k}=q_{\alpha, k}$.

Теорема 1.1 (см. [8]). Пусть $\alpha>-1$ и $\tau>0$. Тогда для любой функиии $f \in B_{2 \tau}$, удовлетворяющей условию

$$
f(x)=O\left(|x|^{-\delta}\right), \quad x \rightarrow \pm \infty, \quad \delta>2 \alpha+2,
$$

справедлива квадратурная формула Бесселя

$$
\int_{0}^{\infty} x^{2 \alpha+1}(f(x)+f(-x)) d x=\frac{2}{\tau^{2 \alpha+2}} \sum_{k=1}^{\infty} \frac{q_{k}^{2 \alpha}}{\left(J_{\alpha}^{\prime}\left(q_{k}\right)\right)^{2}}\left(f\left(\frac{q_{k}}{\tau}\right)+f\left(-\frac{q_{k}}{\tau}\right)\right) .
$$

Формула (1.8) оптимальна в том смъсле, что для любого $\varepsilon>0$ существует функция $f_{\varepsilon} \in B_{2 \tau+\varepsilon}$, удовлетворяющая условию (1.7), для которой равенство (1.8) неверно.

Ясно, что теорему 1.1 достаточно рассмотреть для четных функций $f \in B_{2 \tau}^{0}$. При этом равенство (1.8) приобретает вид (1.2), где

$$
\begin{gathered}
z_{k}=\frac{q_{k}}{\tau}, \quad \gamma_{k}=\frac{2 q_{k}^{2 \alpha}}{\tau^{2 \alpha+2}\left(J_{\alpha}^{\prime}\left(q_{k}\right)\right)^{2}}, \\
z_{k} \sim \frac{\pi}{\tau} k, \quad \gamma_{k} \sim \frac{\pi q_{k}^{2 \alpha+1}}{\tau^{2 \alpha+2}}=\frac{\pi}{\tau} z_{k}^{2 \alpha+1}, \quad k \rightarrow \infty .
\end{gathered}
$$

В работе [8] отмечается идейная связь теоремы 1.1 с классическими формулами Гаусса на отрезке. Кроме того, при $\alpha=-1 / 2$ имеем

$$
J_{-1 / 2}(z)=\sqrt{\frac{2}{\pi z}} \cos z, \quad z_{k}=\frac{\pi(k-1 / 2)}{\tau}, \quad \gamma_{k}=\frac{\pi}{\tau},
$$


и равенство (1.8) может быть получено из классической формулы суммирования Пуассона на оси

$$
\frac{2 \pi}{d} \sum_{k \in \mathbb{Z}} f\left(\frac{x+2 \pi k}{d}\right)=\sum_{n \in \mathbb{Z}} \widehat{f}(d n) e^{i n x}, \quad \widehat{f}(\xi)=\int_{\mathbb{R}} f(x) e^{-i \xi x} d x,
$$

если учесть, что для функции $f \in B_{d}, f=O_{ \pm \infty}\left(|x|^{-1-\varepsilon}\right), \varepsilon>0$, преобразование Фурье $\widehat{f}(\xi)=0$ при $|\xi| \geqslant d$ (следствие теоремы Пэли-Винера; см. [7; гл. IV]).

K. Фрапье и П. Оливье вывели формулу (1.8) методами комплексного анализа. Мы поступим аналогично.

Условие (1.7) теоремы 1.1 можно ослабить. В работе [10] доказано, что теорема справедлива, если

$$
f \in B_{2 \tau}^{0} \cap L_{x^{2 \alpha+1}}^{1}\left(\mathbb{R}_{+}\right)
$$

Позднее изучались варианты приведенных формул по нулям функций Бесселя. В частности, доказаны формулы марковского типа, а также квадратуры с учетом значений производных функции в узлах (см. [11]). Однако аналогичные формулы для весов, отличных от степенного, авторам неизвестны.

Наши основные результаты (теоремы $3.1,4.1$ из $\S 2,3)$ состоят в обобщении теоремы 1.1 с условием (1.10) на случай меры $\sigma$, являющейся спектральной функцией задачи Штурма-Лиувилля (см. [12]). Мы доказываем, что для произвольной функции $f \in B_{2 \tau}^{0} \cap L_{x^{\nu}}^{1}\left(\mathbb{R}_{+}\right)$справедливы квадратурные формулы Гаусса и Маркова

$$
\int_{\mathbb{R}_{+}} f(x) d \sigma(x)=\sum_{k=1}^{\infty} \gamma_{k}(\tau) f\left(z_{k}(\tau)\right)=\sum_{k=0}^{\infty} \gamma_{k}^{\prime}(\tau) f\left(z_{k}^{\prime}(\tau)\right) .
$$

Узлами здесь являются нули собственных функций задач Штурма-Лиувилля, веса положительны, ряды сходятся абсолютно. Формулы оптимальны в том смысле, что существуют допустимые функции типа $>2 \tau$, для которых равенства нарушаются.

Квадратуры Бесселя связаны с интегральным преобразованием Фурье-Ганкеля. Они вкладываются в нашу схему и подробно изложены в $\S 5$. В $\S 5$ рассмотрены и другие важные примеры, отвечающие интегральному преобразованию Якоби и ряду Фурье по ортогональным многочленам Якоби, а также общей задаче Штурма-Лиувилля с регулярным весом.

Основной мотивацией наших исследований являются приложения квадратурных формул для решения экстремальных задач, связанных с гармоническим анализом на многомерных многообразиях. В частности, квадратурные формулы Бесселя применяются в задачах для евклидова пространства $\mathbb{R}^{n}$ (см. [13]-[16]).

Далее, в основном, мы будем работать с нормированной функцией Бесселя (см. [12; гл. V])

$$
j_{\alpha}(z)=2^{\alpha} \Gamma(\alpha+1) z^{-\alpha} J_{\alpha}(z)=F\left(0,0 ; \alpha+1 ;-\frac{z^{2}}{4}\right), \quad \alpha \geqslant-\frac{1}{2},
$$


где $F={ }_{2} F_{1}-$ гипергеометрическая функция Гаусса,

$$
j_{\alpha}(z)=\sum_{k=0}^{\infty} \frac{(-1)^{k} \Gamma(\alpha+1)(z / 2)^{2 k}}{k ! \Gamma(k+\alpha+1)}=\prod_{k=1}^{\infty}\left(1-\frac{z^{2}}{q_{k}^{2}}\right) .
$$

Это четная целая функция экспоненциального типа из класса $B_{1}^{0}, \tau\left(j_{\alpha}\right)=1$. В частности,

$$
j_{-1 / 2}(z)=\cos z, \quad j_{1 / 2}(z)=\frac{\sin z}{z} .
$$

Кроме того,

$$
\left|j_{\alpha}(x)\right| \leqslant j_{\alpha}(0)=1, \quad x \in \mathbb{R}, \quad j_{\alpha}^{\prime}(z)=-\frac{z}{2(\alpha+1)} j_{\alpha+1}(z),
$$

и при $|z| \rightarrow \infty, \operatorname{Re} z \geqslant 0$,

$$
z^{\alpha+1 / 2} j_{\alpha}(z)=A_{\alpha}\left(\cos \left(z-a_{\alpha}\right)+O\left(|z|^{-1} e^{|\operatorname{Im} z|}\right)\right),
$$

где

$$
A_{\alpha}=\frac{2^{\alpha+1 / 2} \Gamma(\alpha+1)}{\sqrt{\pi}}, \quad a_{\alpha}=\frac{\pi(\alpha+1 / 2)}{2} .
$$

В асимптотическом равенстве (1.14) и похожих оценках используется главное значение степенной функции.

\section{§ 2. Задача Штурма-Лиувилля}

Мы рассматриваем меры $\sigma$, которые являются спектральными функциями задач Штурма-Лиувилля (см. [12], [17]-[20]). Это означает, что $\sigma$ участвует в равенстве Парсеваля и теореме разложения по собственным функциям. Отметим, что многие сведения из теории задачи Штурма-Лиувилля в классических трудах приведены в разрозненном виде, что вызывает определенные сложности при их цитировании.

Пусть $T=(0, l), l \leqslant+\infty$, и $w \in C^{1}(T)-$ весовая функция, $w(t)>0, t \in T$. Рассмотрим задачу Штурма-Лиувилля с дифференциальным уравнением

$$
\frac{d}{d t}\left(w(t) \frac{d}{d t} u(t)\right)+z^{2} w(t) u(t)=0, \quad t \in T,
$$

краевыми условиями

$$
u^{\prime}(0)=0, \quad u^{\prime}(l)=0, \quad l<+\infty,
$$

и спектром $\{z\} \subset \mathbb{R}_{+}$. Для $l=+\infty$ он предполагается сплошным $(z \geqslant 0)$ и ему будет отвечать случай дифференцируемой меры $\sigma$ (см. (1.4)). Соответственно при $l<+\infty$ спектр дискретный с нулевым минимальным собственным значением и $\sigma$ - функция скачков (1.5). Для сингулярной задачи в краевых точках обычно требуется ограниченность решения. 
К задаче (2.1), (2.2), которую будем называть стандартной, сводится более общая задача

$$
\begin{gathered}
\left(p v^{\prime}\right)^{\prime}+(\lambda-q) p v=0, \quad p(t)>0, \quad t \in T, \\
v(0) \cos \alpha+v^{\prime}(0) \sin \alpha=0,
\end{gathered}
$$

с аналогичным краевым условием при $t=l<+\infty$ и спектром $\{\lambda\} \subset\left[\lambda_{0},+\infty\right)$.

Действительно, пусть $v_{0}$ - первая собственная функция задачи $(2.3),(2.4)$, отвечающая минимальному собственному значению $\lambda=\lambda_{0}$ :

$$
\left(p v_{0}^{\prime}\right)^{\prime}+\left(\lambda_{0}-q\right) p v_{0}=0 .
$$

Тогда $\left|v_{0}(t)\right| \neq 0, t \in T$ (см. [17; гл. VI]). Применяя стандартную в теории задачи Штурма-Лиувилля формулу

$$
\left(p\left(v^{\prime} v_{0}-v v_{0}^{\prime}\right)\right)^{\prime}+\left(\lambda-\lambda_{0}\right) p v v_{0}=0,
$$

и положив в ней $u=v / v_{0}, w=v_{0}^{2} p, z^{2}=\lambda-\lambda_{0}$, приходим к задаче $(2.1),(2.2)$.

Пусть $\varphi(t, z)$ - решение уравнения $(2.1)$, удовлетворяющее начальным условиям

$$
\varphi(0, z)=1, \quad \varphi_{t}^{\prime}(0, z)=0 .
$$

Ясно, что $\varphi(t, 0)=1, t \in T$.

Если $l<+\infty$, то мера $\sigma$ является функцией скачков $(1.5)$, где $\zeta_{k}-$ собственные значения задачи Штурма-Лиувилля, которые определяются из уравнения $\varphi_{t}^{\prime}(l, z)=0$. Скачки $s_{k}=\left\|\varphi_{\zeta_{k}}\right\|_{2, w}^{-2}$, где $\varphi_{\zeta_{k}}(t)=\varphi\left(t, \zeta_{k}\right)-$ собственные функции задачи Штурма-Лиувилля. В частности, $\zeta_{0}=0, \varphi_{0}=1$ и $s_{0}=\left(\int_{T} w d t\right)^{-1}$. Необходимые асимптотики $\Delta \zeta_{k} \gtrsim 1$ и $s_{k} \asymp \zeta_{k}^{\nu}, k \rightarrow \infty$, обеспечиваются приводимыми далее свойствами функций $\varphi(t, z)$.

При $l=+\infty$ функции $\varphi(t, z)$ также называются собственными функциями задачи Штурма-Лиувилля. Способы построения меры $\sigma$ в этом (сингулярном) случае см. в литературе, приведенной в начале параграфа.

Нам потребуется проверить справедливость следующих свойств функций $\varphi(t, z)$, естественных для теории задачи Штурма-Лиувилля.

1. Аналитичность: для фиксированного $t \in T$ функция $\varphi(t, z) \in B_{t}^{0}$ и

$$
\varphi(t, z)=\prod_{k=1}^{\infty}\left(1-\frac{z^{2}}{z_{k}^{2}(t)}\right), \quad z \in \mathbb{C},
$$

где $0<z_{1}(t)<z_{2}(t)<\cdots-$ положительные нули функции $\varphi(t, z)$ по второму аргументу.

2. Ограниченность: для $t \in T, x \in \mathbb{R}_{+}$

$$
|\varphi(t, x)| \leqslant \varphi(t, 0)=\varphi(0, x)=1 .
$$

3. Асимптотика: равномерно на каждом компакте $\Delta \subset T, t \in \Delta$ (для простоты будем говорить равномерно по $t \in T)$ при $|z| \rightarrow \infty, \operatorname{Re} z \geqslant 0$, имеем

$$
z^{\nu / 2} \varphi(t, z)=C_{t}\left(\cos \left(t z-c_{t}\right)+O\left(|z|^{-1} e^{t|\operatorname{Im} z|}\right)\right),
$$

где $\nu$ - показатель из формулы (1.6). 
4. Равенства Парсеваля: имеем

$$
\|\widehat{f}\|_{2, d \sigma}=\|f\|_{2, w}, \quad\|\widetilde{f}\|_{2, w}=\|f\|_{2, d \sigma}, \quad(f, g)_{w}=(\widehat{f}, \widehat{g})_{d \sigma}, \quad(f, g)_{d \sigma}=(\tilde{f}, \widetilde{g})_{w},
$$

где

$$
\widehat{f}_{w}(z)=\int_{T} f(t) \varphi(t, z) w(t) d t, \quad \widetilde{f}_{d \sigma}(t)=\int_{\mathbb{R}_{+}} f(x) \varphi(t, x) d \sigma(x)
$$

- соответственно прямое и обратное преобразования Фурье функций $f \in L_{w}^{2}(T)$ и $f \in L_{d \sigma}^{2}\left(\mathbb{R}_{+}\right)$по собственным функциям задачи Штурма-Лиувилля.

5. Теорема разложения: если $f \in C(T) \cap L_{w}^{1}(T)$ (тогда $\left.\widehat{f} \in C\left(\mathbb{R}_{+}\right)\right)$и интеграл $\int_{\mathbb{R}_{+}} \widehat{f} \varphi(t, x) d \sigma$ сходится абсолютно и равномерно по $t \in T$, то справедливо поточечное равенство

$$
f(t)=\int_{\mathbb{R}_{+}} \widehat{f}(x) \varphi(t, x) d \sigma(x), \quad t \in T .
$$

Свойства 1-5 достаточно хорошо изучены и выполняются для широкого класса задач Штурма-Лиувилля, например, с регулярным весом $w$ (он положителен в граничных точках). В важных для приложений сингулярных случаях многие необходимые свойства доказаны методами специальных функций и относятся к теории гипергеометрической функции (см. §5).

Прокомментируем указанные свойства. При этом для наглядности будем отмечать соответствующие свойства собственных функций Бесселя $\varphi(t, z)=$ $j_{\alpha}(t z), \alpha \geqslant-1 / 2$. В этом случае (см. [12; гл. $\left.\left.\mathrm{V}, \S 3\right]\right)$

$$
w(t)=\frac{t^{2 \alpha+1}}{2^{2 \alpha} \Gamma^{2}(\alpha+1)}, \quad T=(0,+\infty), \quad s(x)=x^{2 \alpha+1}, \quad\{z\}=\mathbb{R}_{+},
$$

показатель из асимптотики (1.6) $\nu=2 \alpha+1 \geqslant 0$.

В свойстве 1 нули $z_{k}(t)$ являются собственными значениями задачи $\left(w u^{\prime}\right)^{\prime}+$ $z^{2} w u=0$ на интервале $(0, t), u^{\prime}(0)=0, u(t)=0, t \in T$. Они связаны с нулями $t_{k}(z), z \in \mathbb{R}_{+}$, уравнения $\varphi(t, z)=0$ равенством $t_{k}\left(z_{k}(t)\right)=t$ или $z_{k}(t)=t_{k}^{-1}(t)$. Из теории Штурма известно, что нули $t_{k}(z)$ непрерывны и монотонно убывают к нулю при возрастании $z$ (см. [17; гл. VI], [19; гл. I]). Поэтому нули $z_{k}(t)$ также непрерывны и монотонно убывают к нулю при возрастании $t$. При этом из асимптотики собственных функций (2.7) для $\operatorname{Im} z=0$ получаем

$$
z_{k}(t)=\frac{\pi(k-1 / 2)+c_{t}}{t}+O\left(k^{-1}\right), \quad k \rightarrow \infty .
$$

Для собственных функций Бесселя имеем $t_{k}(z)=q_{k} / z$ и $z_{k}(t)=q_{k} / t$, где $q_{k} \sim \pi k$.

Свойства 1, 2 частично вытекают из интегрального представления Мелера

$$
\varphi(t, z)=\int_{0}^{t} \cos (z u) d \mu_{t}(u)
$$

где $d \mu_{t} \geqslant 0$. В этом случае принадлежность классу $B_{t}^{0}$ очевидна и

$$
|\varphi(t, x)| \leqslant \int_{0}^{t} d \mu_{t}=1, \quad x \in \mathbb{R}_{+}, \quad t \in T .
$$


Для функций Бесселя при $\alpha>-1 / 2$

$$
j_{\alpha}(t z)=\frac{2 C_{\alpha}}{t^{2 \alpha}} \int_{0}^{t}\left(t^{2}-u^{2}\right)^{\alpha-1 / 2} \cos (z u) d u
$$

где

$$
C_{\alpha}=\frac{\Gamma(\alpha+1)}{\sqrt{\pi} \Gamma(\alpha+1 / 2)} .
$$

Неравенство (2.6) также выполняется, если вес $w$ не убывает (см. [2; гл. VII]). В этом случае для функции $h(t)=z^{2} u^{2}+\left(u^{\prime}\right)^{2}$ из уравнения $(2.1)$ имеем $h^{\prime}=\left(w^{-2}\right)^{\prime} w^{2}\left(u^{\prime}\right)^{2} \leqslant 0$ и $h$ не возрастает. Следовательно, последовательные максимумы $u^{2}\left(t_{i}\right)$, равные $z^{-2} h\left(t_{i}\right)\left(u^{\prime}\left(t_{i}\right)=0\right)$, не возрастают и $|u(t)| \leqslant u(0)$, $t \in T$.

Вес Бесселя $w(t)=2^{-2 \alpha} \Gamma^{-2}(\alpha+1) t^{2 \alpha+1}$ не убывает, если $\alpha \geqslant-1 / 2$.

Функции $\varphi(t, z)$ по переменному $z$ аналитичны во всей комплексной области и четны. По переменному $t$ аналитичность можно гарантировать только в окрестности интервала $T$. Отсюда, следуя [21; гл. 1], из асимптотического равенства (2.7) (свойство 3) находим, что при тех же $z$ и $j=0,1,2, \ldots$

$$
\begin{aligned}
& z^{\nu / 2} \varphi_{z}^{(j)}(t, z)=t^{j} C_{t}\left(\cos \left(t z-c_{t}+\frac{\pi j}{2}\right)+O\left(|z|^{-1} e^{t|\operatorname{Im} z|}\right)\right. \\
& z^{\nu / 2} \varphi_{t}^{(j)}(t, z)=z^{j} C_{t}\left(\cos \left(t z-c_{t}+\frac{\pi j}{2}\right)+O\left(|z|^{-1} e^{t|\operatorname{Im} z|}\right)\right)
\end{aligned}
$$

Для функций Бесселя асимптотики (2.7), (2.12), (2.13) вытекают из формулы (1.14). При этом можно независимо использовать формулу дифференцирования (1.13).

Отметим, что во всех наших примерах $c_{t}=$ const и

$$
z^{-\nu / 2} C_{t} \sim \frac{(2 / \pi)^{1 / 2}}{(w(t) s(z))^{1 / 2}}, \quad|z| \rightarrow \infty, \quad t \in T
$$

Причем это верно и в дискретных случаях, если определить вес $s(x) \asymp x^{\nu}$ так, чтобы $s\left(\zeta_{k}\right) \sim s_{k}$.

Доказательства полноты собственных функций, равенства Парсеваля и теоремы разложения (свойства 4,5 ) являются одними из основных выводов теории задачи Штурма-Лиувилля. Обычно вначале они доказываются на плотных классах гладких функций. В наших примерах (см. §5) свойства 4 и 5 справедливы.

Отметим, что в силу (2.7) имеем $\varphi(t, x)=O_{+\infty}\left(x^{-\nu / 2}\right), t \in \Delta \subset T$. Поэтому для абсолютной и равномерной сходимости интеграла $\int_{\mathbb{R}_{+}} \widehat{f} \varphi(t, x) d \sigma$ достаточ-

Случаю веса Бесселя отвечает преобразование Фурье-Ганкеля (см. [12]), для которого свойства 4,5 хорошо известны. При $\alpha=-1 / 2$ и $1 / 2$ в силу (1.12) оно сводится к косинус- и синус-преобразованиям Фурье соответственно. 


\section{§ 3. Квадратурная формула Гаусса}

Перейдем к формулировке основного результата. Зафиксируем $\tau \in T$, и пусть везде далее

$$
z_{k}=z_{k}(\tau), \quad \varphi(z)=\varphi(\tau, z), \quad \varphi^{\prime}(z)=\varphi_{z}^{\prime}(\tau, z) .
$$

Нули $z_{k}$ будут узлами конструируемой квадратурной формулы Гаусса.

Для $k \in \mathbb{N}$ введем функции

$$
\Phi_{k}(z)=\Phi_{k}(\tau, z)=\frac{2 z_{k} \varphi(z)}{\varphi^{\prime}\left(z_{k}\right)\left(z^{2}-z_{k}^{2}\right)} \in B_{\tau}^{0} .
$$

Таким образом, $\Phi_{k}\left(z_{j}\right)=\delta_{k j}$. В силу $(2.7)$ имеем

$$
\Phi_{k}(x)=O\left(x^{-\nu / 2-2}\right), \quad x \rightarrow+\infty .
$$

Поэтому по условию на меру (1.6) функции $\Phi_{k}^{2} \in L_{d \sigma}^{1}\left(\mathbb{R}_{+}\right)$и можно положить

$$
\gamma_{k}=\gamma_{k}(\tau)=\int_{\mathbb{R}_{+}} \Phi_{k}^{2}(\tau, x) d \sigma(x) .
$$

Это будут веса квадратурной формулы. Другое выражение для них дается ниже в лемме 3.2 .

Сформулируем основной результат для меры $\sigma$, удовлетворяющей вышеперечисленным условиям. Пусть $T / 2=(0, l / 2)$ при $l<+\infty$ и $T / 2=(0,+\infty)$ при $l=+\infty$.

ТеОрема 3.1. Для $\tau \in T / 2$ и произвольной функиии $f \in B_{2 \tau}^{0} \cap L_{x^{\nu}}^{1}\left(\mathbb{R}_{+}\right)$ справедлива квадратурная формула Гаусса

$$
\int_{\mathbb{R}_{+}} f(x) d \sigma(x)=\sum_{k=1}^{\infty} \gamma_{k} f\left(z_{k}\right) .
$$

Ряд в (3.4) сходится абсолютно.

Формула (3.4) оптимальна в том смысле, что для любого в больше нуля существует допустимая функиия $f_{\varepsilon} \in B_{2 \tau+\varepsilon}^{0} \cap L_{x^{\nu}}^{1}\left(\mathbb{R}_{+}\right)$, для которой равенство (3.4) неверно.

ЗАмЕчАниЕ 3.1. Согласно асимптотике (2.8) плотность узлов $z_{k}$ из квадратурной формулы (3.4) равна

$$
\delta=\lim _{k \rightarrow \infty} \frac{k}{z_{k}}=\frac{\tau}{\pi} .
$$

Было бы интересно показать, что никакая квадратурная формула с плотностью узлов $\tau / \pi$ не может быть точной для всех четных целых функций экспоненциального типа $>2 \tau$. Это доказывало бы оптимальный характер квадратурной формулы (3.4) в терминах плотности узлов и точности на целых функциях экспоненциального типа. 
Доказательство теоремы 3.1 разобьем на ряд вспомогательных утверждений. Вначале, следуя работе [8; лемма 3], выведем вспомогательную интерполяционную формулу.

Лемма 3.1. Для функиий $f \in B_{2 \tau}^{0}$, удовлетворяющих условию

$$
f(x)=O\left(x^{-\delta}\right), \quad x \rightarrow+\infty, \quad \delta>\nu+1,
$$

на любом компакте из $\mathbb{C}$ справедливо поточечное равенство

$$
f(z)=\sum_{k=1}^{\infty}\left(A_{k}(z) f\left(z_{k}\right)+B_{k}(z) f^{\prime}\left(z_{k}\right)\right), \quad z \in \mathbb{C},
$$

где

$$
A_{k}(z)=\left(1+\left(\frac{1}{z_{k}}-\frac{\varphi^{\prime \prime}\left(z_{k}\right)}{\varphi^{\prime}\left(z_{k}\right)}\right) \frac{z^{2}-z_{k}^{2}}{2 z_{k}}\right) \Phi_{k}^{2}(z), \quad B_{k}(z)=\frac{z^{2}-z_{k}^{2}}{2 z_{k}} \Phi_{k}^{2}(z) .
$$

ДокАзАтельство. В работе [8; леммы 1, 2] показано, что:

1) равномерно по $|\theta| \leqslant \pi$

$$
\begin{gathered}
f\left(r e^{i \theta}\right)=O\left(\frac{e^{2 \tau r|\sin \theta|}}{r^{\delta}}\right), \quad r \rightarrow+\infty \\
f^{\prime}(z)=O\left(z^{-\delta}\right), \quad z \rightarrow+\infty
\end{gathered}
$$

2) для $|\theta| \leqslant \pi$

$$
\left|\cos \left(R e^{i \theta}-c\right)\right| \geqslant K e^{R|\sin \theta|},
$$

где $K>0, R=\pi N+c, N-$ большое целое число.

Отсюда, из асимптотики (2.7) и четности функции $\varphi$ следует, что для $r=$ $R / \tau, c=c_{\tau}$

$$
r^{\nu / 2}\left|\varphi\left(r e^{i \theta}\right)\right| \gtrsim\left|\cos \left(R e^{i \theta}-c\right)\right|+O\left(r^{-1} e^{\tau r|\sin \theta|}\right) \gtrsim e^{\tau r|\sin \theta|}\left(1+O\left(r^{-1}\right)\right) .
$$

Рассмотрим контурный интеграл

$$
I_{N}(z)=\frac{1}{2 \pi i} \oint_{|\zeta|=r} \frac{f(\zeta)}{(\zeta-z) \varphi^{2}(\zeta)} d \zeta
$$

где $r=(\pi N+c) / \tau, z \in \mathbb{C},|z|<r$. При $z \in\left\{z_{k}\right\}$ формула (3.6) очевидна, поэтому можно считать, что $z \notin\left\{z_{k}\right\}$.

Имеем

$$
\left|I_{N}\right| \leqslant \frac{1}{2 \pi} \int_{-\pi}^{\pi} \frac{r\left|f\left(r e^{i \theta}\right)\right|}{\left|r e^{i \theta}-z\right|\left|\varphi\left(r e^{i \theta}\right)\right|^{2}} d \theta \lesssim r^{-\delta+\nu} .
$$

Поскольку $\delta>\nu+1$, то $-\delta+\nu<-1$ и $I_{N}(z) \rightarrow 0$ при $N \rightarrow \infty$ равномерно по $z$ на любом компакте из $\mathbb{C}$.

С другой стороны, учитывая положение полюсов интегрируемой функции, имеем

$$
I_{N}=\operatorname{Res}_{z}+\sum_{\left|z_{k}\right|<r} \operatorname{Res}_{z_{k}}
$$

где $z_{k}=-z_{-k}$ при $k<0$. 
Найдем соответствующие вычеты. В точке $\zeta=z$ имеем простой полюс и

$$
\operatorname{Res}_{z} \frac{f(\zeta)}{(\zeta-z) \varphi^{2}(\zeta)}=\frac{f(z)}{\varphi^{2}(z)}
$$

В нулях $\zeta=z_{k}$ функции $\varphi-$ полюсы второго порядка. Поэтому

$$
\operatorname{Res}_{z_{k}}=\lim _{\zeta \rightarrow z_{k}} \frac{d}{d \zeta} \frac{\left(\zeta-z_{k}\right)^{2} f(\zeta)}{(\zeta-z) \varphi^{2}(\zeta)}
$$

Имеем

$$
\left(\frac{f}{\zeta-z}\left(\frac{\zeta-z_{k}}{\varphi}\right)^{2}\right)^{\prime}=\left(\frac{f}{\zeta-z}\right)^{\prime}\left(\frac{\zeta-z_{k}}{\varphi}\right)^{2}+2 \frac{f}{\zeta-z} \frac{\zeta-z_{k}}{\varphi}\left(\frac{\zeta-z_{k}}{\varphi}\right)^{\prime}
$$

Здесь

$$
\begin{gathered}
\lim _{\zeta \rightarrow z_{k}}\left(\frac{f}{\zeta-z}\right)^{\prime}=\frac{f^{\prime}\left(z_{k}\right)\left(z_{k}-z\right)-f\left(z_{k}\right)}{\left(z_{k}-z\right)^{2}} \\
\lim _{\zeta \rightarrow z_{k}} \frac{\zeta-z_{k}}{\varphi}=\frac{1}{\varphi^{\prime}\left(z_{k}\right)}, \quad \lim _{\zeta \rightarrow z_{k}} \frac{f}{\zeta-z}=\frac{f\left(z_{k}\right)}{z_{k}-z}, \\
\lim _{\zeta \rightarrow z_{k}}\left(\frac{\zeta-z_{k}}{\varphi}\right)^{\prime}=\lim _{\zeta \rightarrow z_{k}} \frac{\varphi-\left(\zeta-z_{k}\right) \varphi^{\prime}}{\varphi^{2}}=\lim _{\zeta \rightarrow z_{k}} \frac{-\left(\zeta-z_{k}\right) \varphi^{\prime \prime}}{2 \varphi \varphi^{\prime}}=\frac{-\varphi^{\prime \prime}\left(z_{k}\right)}{2\left(\varphi^{\prime}\right)^{2}\left(z_{k}\right)} .
\end{gathered}
$$

Таким образом,

$$
\operatorname{Res}_{z_{k}}=\left(\frac{f^{\prime}\left(z_{k}\right)}{z_{k}-z}-\frac{f\left(z_{k}\right)}{\left(z_{k}-z\right)^{2}}\right) \frac{1}{\left(\varphi^{\prime}\right)^{2}\left(z_{k}\right)}+\frac{f\left(z_{k}\right)}{z_{k}-z} \frac{-\varphi^{\prime \prime}\left(z_{k}\right)}{\left(\varphi^{\prime}\right)^{3}\left(z_{k}\right)},
$$

или

$$
\operatorname{Res}_{z_{k}}=-a_{k} f\left(z_{k}\right)-b_{k} f^{\prime}\left(z_{k}\right)
$$

где

$$
a_{k}=\frac{\left(\varphi^{\prime}\right)^{-2}\left(z_{k}\right)}{\left(z-z_{k}\right)^{2}}-\frac{\varphi^{\prime \prime}\left(z_{k}\right)\left(\varphi^{\prime}\right)^{-3}\left(z_{k}\right)}{z-z_{k}}, \quad b_{k}=\frac{\left(\varphi^{\prime}\right)^{-2}\left(z_{k}\right)}{z-z_{k}} .
$$

Собирая полученные результаты вместе, получаем

$$
\frac{f}{\varphi^{2}}+\sum_{k \in \mathbb{Z} \backslash\{0\}} \operatorname{Res}_{z_{k}}=0 .
$$

Отсюда, используя четность функции $f$, нечетность функции $f^{\prime}$ и равенство $z_{-k}=-z_{k}$, находим

$$
f=\varphi^{2} \sum_{k=1}^{\infty}\left(\left(a_{k}+a_{-k}\right) f\left(z_{k}\right)+\left(b_{k}-b_{-k}\right) f^{\prime}\left(z_{k}\right)\right) .
$$

В силу четности функции $\varphi$ имеем

$$
a_{k}+a_{-k}=\frac{\left(\varphi^{\prime}\right)^{-2}\left(z_{k}\right)}{\left(z-z_{k}\right)^{2}}-\frac{\varphi^{\prime \prime}\left(z_{k}\right)\left(\varphi^{\prime}\right)^{-3}\left(z_{k}\right)}{z-z_{k}}+\frac{\left(\varphi^{\prime}\right)^{-2}\left(z_{k}\right)}{\left(z+z_{k}\right)^{2}}+\frac{\varphi^{\prime \prime}\left(z_{k}\right)\left(\varphi^{\prime}\right)^{-3}\left(z_{k}\right)}{z+z_{k}},
$$


откуда

$$
A_{k}=\left(a_{k}+a_{-k}\right) \varphi^{2}=\left(\frac{2\left(z^{2}+z_{k}^{2}\right)}{\left(\varphi^{\prime}\right)^{2}\left(z_{k}\right)\left(z^{2}-z_{k}^{2}\right)^{2}}-\frac{2 z_{k} \varphi^{\prime \prime}\left(z_{k}\right)}{\left(\varphi^{\prime}\right)^{3}\left(z_{k}\right)\left(z^{2}-z_{k}^{2}\right)}\right) \varphi^{2} .
$$

Аналогично

$$
B_{k}=\left(b_{k}-b_{-k}\right) \varphi^{2}=\frac{2 z_{k}}{\left(\varphi^{\prime}\right)^{2}\left(z_{k}\right)\left(z^{2}-z_{k}^{2}\right)} \varphi^{2} .
$$

В терминах функции (3.1) имеем

$$
A_{k}=\left(1+\left(\frac{1}{z_{k}}-\frac{\varphi^{\prime \prime}\left(z_{k}\right)}{\varphi^{\prime}\left(z_{k}\right)}\right) \frac{z^{2}-z_{k}^{2}}{2 z_{k}}\right) \Phi_{k}^{2}, \quad B_{k}=\frac{z^{2}-z_{k}^{2}}{2 z_{k}} \Phi_{k}^{2} .
$$

Лемма 3.1 доказана.

В качестве примера рассмотрим случай веса Бесселя при $\tau=1$. Тогда

$$
\varphi(z)=j_{\alpha}(z), \quad z_{k}=q_{k}, \quad\left(z^{\nu} \varphi^{\prime}\right)^{\prime}+z^{\nu} \varphi=0,
$$

где $\nu=2 \alpha+1$. Отсюда следует, что $z_{k} \varphi^{\prime \prime}\left(z_{k}\right)=-\nu \varphi^{\prime}\left(z_{k}\right)$ и

$$
A_{k}=\left(1+\frac{(\nu+1)\left(z^{2}-z_{k}^{2}\right)}{2 z_{k}^{2}}\right) \Phi_{k}^{2} .
$$

В терминах функции Бесселя $J_{\alpha}$ после несложных преобразований находим

$$
\begin{gathered}
\Phi_{k}(z)=\frac{2 q_{k}^{\alpha+1} J_{\alpha}(z)}{J_{\alpha}^{\prime}\left(q_{k}\right) z^{\alpha}\left(z^{2}-q_{k}^{2}\right)}, \\
A_{k}(z)=\frac{q_{k}^{2 \alpha} J_{\alpha}^{2}(z)}{\left(J_{\alpha}^{\prime}\left(q_{k}\right)\right)^{2} z^{2 \alpha}}\left(\frac{1}{\left(z-q_{k}\right)^{2}}+\frac{1}{\left(z+q_{k}\right)^{2}}+\frac{2(2 \alpha+1)}{z^{2}-q_{k}^{2}}\right), \\
B_{k}(z)=\frac{2 q_{k}^{2 \alpha+1} J_{\alpha}^{2}(z)}{\left(J_{\alpha}^{\prime}\left(q_{k}\right)\right)^{2} z^{2 \alpha}\left(z^{2}-q_{k}^{2}\right)} .
\end{gathered}
$$

Эти выражения совпадают с выражениями, приведенными в [8; (45)].

Далее для $k \in \mathbb{N}$ положим

$$
\varphi_{k}(t)=\varphi\left(t, z_{k}\right), \quad \varphi_{k}^{\prime}(t)=\varphi_{t}^{\prime}\left(t, z_{k}\right), \quad v_{k}(t)=\gamma_{k} \varphi_{k}(t) \chi_{[0, \tau]}(t),
$$

где $\chi_{I}$ - характеристическая функция множества $I$.

Имеем $v_{k}(\tau-0)=\gamma_{k} \varphi\left(\tau, z_{k}\right)=0$, поэтому $v_{k} \in C(T)$. Также очевидно, что $v_{k} \in L_{w}^{1}(T)$.

Лемма 3.2. Пусть $k \in \mathbb{N}$. Функиия $v_{k}$ u ее преобразование Фуръе $\widehat{v}_{k}$ удовлетворяют условиям теоремы разложения и

$$
\widehat{v}_{k}=\Phi_{k}, \quad \widetilde{\Phi}_{k}=v_{k} .
$$

В частности,

$$
\int_{\mathbb{R}_{+}} \Phi_{k}(x) \varphi(x) d \sigma(x)=\widetilde{\Phi}_{k}(\tau)=v_{k}(\tau)=0 .
$$


Кроме того, справедливы соотношения ортогональности

$$
\left(v_{j}, v_{k}\right)_{w}=\left(\Phi_{j}, \Phi_{k}\right)_{d \sigma}=\gamma_{j} \delta_{j k}
$$

и формула для весов

$$
\gamma_{k}=\left\|\varphi_{k}\right\|_{L_{w}^{2}(0, \tau)}^{-2}=\frac{2 z_{k}}{w(\tau) \varphi_{k}^{\prime}(\tau) \varphi^{\prime}\left(z_{k}\right)}
$$

ДокАзАТЕльство. Так как $v_{k} \in C(T) \cap L_{w}^{1}(T)$ и справедливо (3.2), то теорема разложения будет выполнена, если показать, что $\widehat{v}_{k}=\Phi_{k}$.

Пусть $u_{1}, u_{2}$ - решения задачи Штурма-Лиувилля, соответствующие значениям $z_{1}, z_{2}$. Тогда по аналогии с равенством (2.5) имеем

$$
\left(w\left(u_{1}^{\prime} u_{2}-u_{1} u_{2}^{\prime}\right)\right)^{\prime}+\left(z_{1}^{2}-z_{2}^{2}\right) w u_{1} u_{2}=0 .
$$

Отсюда следует, что

$$
\int_{0}^{\tau} u_{1} u_{2} w d t=-\left.\frac{w\left(u_{1}^{\prime} u_{2}-u_{1} u_{2}^{\prime}\right)}{z_{1}^{2}-z_{2}^{2}}\right|_{t=0} ^{\tau}=\frac{w(\tau)\left(u_{1}^{\prime}(\tau) u_{2}(\tau)-u_{1}(\tau) u_{2}^{\prime}(\tau)\right)}{z_{2}^{2}-z_{1}^{2}} .
$$

Полагая $z_{1}=z_{k}, z_{2}=z, u_{1}=\varphi\left(t, z_{k}\right), u_{2}=\varphi(t, z)$ и пользуясь принятыми обозначениями, получаем

$$
\int_{0}^{\tau} \varphi_{k} \varphi(t, z) w d t=\frac{w(\tau) \varphi_{k}^{\prime}(\tau) \varphi(z)}{z^{2}-z_{k}^{2}}=d_{k}^{-1} \Phi_{k}(z)
$$

где

$$
d_{k}=\frac{2 z_{k}}{w(\tau) \varphi_{k}^{\prime}(\tau) \varphi^{\prime}\left(z_{k}\right)}
$$

Отсюда следует, что

$$
\widehat{v}_{k}=\gamma_{k} d_{k}^{-1} \Phi_{k}, \quad \widetilde{\Phi}_{k}=d_{k} \gamma_{k}^{-1} v_{k}
$$

Кроме того, при $z=z_{j}$

$$
d_{k} \int_{0}^{\tau} \varphi_{k} \varphi_{j} w d t=\Phi_{k}\left(z_{j}\right)=\delta_{k j}
$$

Покажем, что $d_{k}=\gamma_{k}$. Воспользуемся равенством Парсеваля:

$$
\left(\Phi_{j}, \Phi_{k}\right)_{d \sigma}=\left(\widetilde{\Phi}_{j}, \widetilde{\Phi}_{k}\right)_{w}=d_{j} \gamma_{j}^{-1} d_{k} \gamma_{k}^{-1}\left(v_{j}, v_{k}\right)_{w}=d_{j} d_{k} \int_{0}^{\tau} \varphi_{j} \varphi_{k} w d t=d_{j} \delta_{j k}
$$

откуда, используя определение (3.3), находим $\gamma_{k}=\left(\Phi_{k}, \Phi_{k}\right) d \sigma=d_{k}$.

Таким образом, $\widehat{v}_{k}=\Phi_{k}$ и поточечно при $t \in T$

$$
\widetilde{\Phi}_{k}(t)=\int_{\mathbb{R}_{+}} \Phi_{k}(x) \varphi(t, x) d \sigma(x)=v_{k}(t) .
$$

Лемма доказана. 
Заметим, что функции $v_{k}$ являются собственными функциями задачи Штурма-Лиувилля на интервале $(0, \tau)$ с дифференциальным уравнением $(2.1)$, краевыми условиями $u^{\prime}(0)=1, u(\tau)=0$ и собственными значениями $z_{k}$. Они образуют полную ортогональную систему в пространстве $L_{w}^{2}(0, \tau)$ (см. [12]).

Выведем асимптотику весов $\gamma_{k}$.

ЛЕмма 3.3. При $k \rightarrow \infty$

$$
\gamma_{k} \asymp z_{k}^{\nu} .
$$

ДокАЗАТЕЛЬСтво. По лемме 3.2 имеем

$$
\gamma_{k}=\frac{2 z_{k}}{w(\tau) \varphi_{k}^{\prime}(\tau) \varphi^{\prime}\left(z_{k}\right)}, \quad k \in \mathbb{N} .
$$

Воспользуемся асимптотиками $(2.8)$ и $(2.12),(2.13)$ для $j=1$. Тогда при $k \rightarrow \infty$

$$
\begin{gathered}
\varphi_{z}^{\prime}\left(\tau, z_{k}\right) \sim z_{k}^{-\nu / 2} \tau C_{\tau}\left\{-\sin \left(\pi\left(k-\frac{1}{2}\right)+O\left(k^{-1}\right)\right)\right\} \sim(-1)^{k+1} z_{k}^{-\nu / 2} \tau C_{\tau} \\
\varphi_{t}^{\prime}\left(\tau, z_{k}\right) \sim z_{k}^{-\nu / 2+1} C_{\tau}\left\{-\sin \left(\pi\left(k-\frac{1}{2}\right)+O\left(k^{-1}\right)\right)\right\} \sim(-1)^{k+1} z_{k}^{-\nu / 2+1} C_{\tau} .
\end{gathered}
$$

Таким образом, $\varphi_{k}^{\prime}(\tau) \varphi^{\prime}\left(z_{k}\right) \sim z_{k}^{-\nu+1} \tau C_{\tau}^{2}$ и $\gamma_{k} \asymp z_{k}^{\nu}$.

Лемма доказана.

Из лемм 3.1, 3.2 вытекает квадратурная формула Гаусса при выполнении условия (3.5). Она является аналогом теоремы 1.1.

Лемма 3.4. Если функиия $f \in B_{2 \tau}^{0}$ удовлетворяет оценке (3.5), то справедлива квадратурная формула (3.4).

ДокАзАтЕльство. Достаточно проинтегрировать с мерой $\sigma$ интерполяционную формулу (3.6) из леммы 3.1 и воспользоваться определением весов (3.3) и равенством (3.9) из леммы 3.2 :

$$
\int_{\mathbb{R}_{+}} \Phi_{k}^{2} d \sigma=\gamma_{k}, \quad \int_{\mathbb{R}_{+}}\left(x^{2}-z_{k}^{2}\right) \Phi_{k}^{2} d \sigma=\frac{2 z_{k}}{\varphi^{\prime}\left(z_{k}\right)} \int_{\mathbb{R}_{+}} \Phi_{k} \varphi d \sigma=0 .
$$

Для обоснования возможности почленного интегрирования докажем, что ряд в равенстве (3.6) сходится в пространстве $L_{d \sigma}^{1}\left(\mathbb{R}_{+}\right)$.

Оценим $\left\|B_{k}\right\|_{1, d \sigma}$. Из определения функции $B_{k}$ и равенства

$$
\int_{\mathbb{R}_{+}} \frac{\varphi^{2}}{x^{2}-z_{k}^{2}} d \sigma=\frac{\varphi^{\prime}\left(z_{k}\right)}{2 z_{k}} \int_{\mathbb{R}_{+}} \Phi_{k} \varphi d \sigma=0
$$

находим

$$
\int_{\mathbb{R}_{+}}\left|B_{k}\right| d \sigma \leqslant \frac{2 z_{k}}{\left(\varphi^{\prime}\right)^{2}\left(z_{k}\right)} \int_{\mathbb{R}_{+}} \frac{\varphi^{2}}{\left|x^{2}-z_{k}^{2}\right|} d \sigma=\frac{4 z_{k}}{\left(\varphi^{\prime}\right)^{2}\left(z_{k}\right)} \int_{z_{k}}^{\infty} \frac{\varphi^{2}}{x^{2}-z_{k}^{2}} d \sigma \lesssim z_{k}^{\nu+1} I,
$$

где

$$
I=\int_{z_{k}}^{\infty} \frac{\varphi^{2}}{x^{2}-z_{k}^{2}} d \sigma=\int_{z_{k}}^{z_{k}+z_{1}}+\int_{z_{k}+z_{1}}^{3 z_{k}}+\int_{3 z_{k}}^{\infty}=I_{1}+I_{2}+I_{3}
$$


Для третьего слагаемого имеем

$$
I_{3} \lesssim \int_{3 z_{k}}^{\infty} \frac{\varphi^{2}}{x^{2}} d \sigma \lesssim \int_{3 z_{k}}^{\infty} \frac{d x}{x^{2}} \lesssim z_{k}^{-1}
$$

где использована оценка $|\varphi(x)| \lesssim x^{-\nu / 2}, x \gtrsim 1$. Аналогично, для второго слагаемого

$$
I_{2} \lesssim \int_{z_{k}+z_{1}}^{3 z_{k}} \frac{\varphi^{2}}{\left(x-z_{k}\right)\left(x+z_{k}\right)} d \sigma \lesssim \frac{1}{z_{k}} \int_{z_{k}+z_{1}}^{3 z_{k}} \frac{d x}{x-z_{k}} \lesssim z_{k}^{-1} \ln z_{k} .
$$

По теореме Лагранжа о среднем значении и асимптотике для производной (2.12) находим

$$
\frac{\varphi^{2}(z)}{x^{2}-z_{k}^{2}}=\frac{\varphi}{x+z_{k}} \varphi^{\prime}(\theta)=O\left(z_{k}^{-\nu-1}\right), \quad \theta \in\left(z_{k}, z_{k}+z_{1}\right) .
$$

Поэтому для первого слагаемого имеем

$$
I_{1} \lesssim z_{k}^{-\nu-1} \int_{z_{k}}^{z_{k}+z_{1}} x^{\nu} d x \lesssim z_{k}^{-1}
$$

Собирая оценки вместе, получаем

$$
\left\|B_{k}\right\|_{1, d \sigma} \lesssim z_{k}^{\nu+1} z_{k}^{-1} \ln z_{k}=z_{k}^{\nu} \ln z_{k}
$$

Теперь оценим $\left\|A_{k}\right\|_{1, d \sigma}$. Из оценок $\left|\varphi_{z}^{\prime}\left(z_{k}\right)\right| \asymp z_{k}^{-\nu / 2}$ и $\left|\varphi_{z}^{\prime \prime}\left(z_{k}\right)\right| \lesssim z_{k}^{-\nu / 2}$ имеем $\left|\varphi^{\prime \prime}\left(z_{k}\right) / \varphi^{\prime}\left(z_{k}\right)\right| \lesssim 1$. Отсюда, из определения (3.3) и леммы 3.3 следует, что

$$
\int_{\mathbb{R}_{+}}\left|A_{k}\right| d \sigma \lesssim \int_{\mathbb{R}_{+}} \Phi_{k}^{2} d \sigma+\int_{\mathbb{R}_{+}}\left|B_{k}\right| d \sigma=\gamma_{k}+\left\|B_{k}\right\|_{1, d \sigma} \lesssim z_{k}^{\nu} \ln z_{k} .
$$

Наконец, воспользуемся оценкой (3.5), вытекающей из нее оценкой (3.7) и асимптотикой $z_{k} \asymp k$. Тогда $\forall z \in \mathbb{R}_{+}$имеем

$$
\sum_{k=1}^{\infty}\left(\left|f\left(z_{k}\right)\right| \int_{\mathbb{R}_{+}}\left|A_{k}(x)\right| d \sigma+\left|f^{\prime}\left(z_{k}\right)\right| \int_{\mathbb{R}_{+}}\left|B_{k}(x)\right| d \sigma\right) \lesssim \sum_{k=1}^{\infty} z_{k}^{\nu-\delta} \ln z_{k}<\infty
$$

если $\nu-\delta<-1$.

Лемма 3.4 доказана.

Заменим условие (3.5) более общим $\|f\|_{1, x^{\nu}}<\infty$. Основную сложность представляет случай дискретной меры $\sigma$. Для этого случая докажем вариант известного неравенства Планшереля-Полиа (см. [7; дополнения и задачи II]) (случай $\nu=0)$.

Лемма 3.5. Пусть $\xi_{0}=0<\xi_{1}<\xi_{2}<\cdots, a_{k} \geqslant 0, k \in \mathbb{Z}_{+}$,

$$
\inf _{k \in \mathbb{Z}_{+}} \Delta \xi_{k}>0, \quad a_{k}=O\left(\xi_{k}^{\nu}\right), \quad k \rightarrow \infty
$$


где $\nu \geqslant 0$. Тогда для любой четной или нечетной функиии $f \in B_{d} \cap L_{x^{\nu}}^{1}\left(\mathbb{R}_{+}\right)$, $d>0$, справедлива оченка

$$
\sum_{k=0}^{\infty} a_{k}\left|f\left(\xi_{k}\right)\right| \lesssim(1+d) \int_{\mathbb{R}_{+}}|f(x)| x^{\nu} d x
$$

где константа в неравенстве не зависит от типа $d$.

ДокАЗАТЕЛьство. Определим неотрицательную на $\mathbb{R}$ функцию $\omega \in B_{2}^{0}$, для которой $\omega(x) \asymp x^{\nu}$ при $x \rightarrow+\infty$. Для этого представим показатель $\nu \geqslant 0$ в виде $\nu=2 n-(2 \alpha+1)$, где $n \in \mathbb{N},|\alpha| \leqslant 1 / 2(\alpha=0$, если $\nu=2 n-1)$, и положим

$$
\omega(z)=z^{2 n} j_{\alpha}(z+i) j_{\alpha}(z-i) .
$$

Имеем $\tau(\omega)=2$ и в силу (1.14) при $x \rightarrow+\infty$

$$
\omega(x) \asymp x^{2 n} x^{-2 \alpha-1}\left(\cos (x+i-a) \cos (x-i-a)+O\left(x^{-1}\right)\right)
$$

или

$$
\omega(x) \asymp x^{\nu}\left(\operatorname{ch}^{2} 1 \cos ^{2}(x-a)+\operatorname{sh}^{2} 1 \sin ^{2}(x-a)+O\left(x^{-1}\right)\right) \asymp x^{\nu} .
$$

Отсюда и из того, что все нули функции $\omega$, кроме $z=0$, лежат на прямых $\operatorname{Im} z= \pm 1$, следует, что $\omega(x)>0$ при $|x|>0$.

Пусть $f$ - допустимая функция и положим $g=f \omega$. Тогда функция $g \in B_{d+2}$ и имеет ту же четность, что и $f$, поэтому $|g(x)|=|g(-x)|,\left|g^{\prime}(x)\right|=\left|g^{\prime}(-x)\right|$. Отсюда и из оценки $\omega(x) \lesssim x^{\nu}$, верной для любого $x \in \mathbb{R}_{+}$, имеем

$$
\|g\|_{L^{1}(\mathbb{R})}=2 \int_{\mathbb{R}_{+}}|g(x)| d x \lesssim \int_{\mathbb{R}_{+}}|f(x)| x^{\nu} d x<\infty .
$$

Следуя [22; гл. 3] покажем, что

$$
S=\sum_{k=0}^{\infty}\left|g\left(\xi_{k}\right)\right| \lesssim\|g\|_{L^{1}(\mathbb{R})}
$$

По теореме о среднем значении имеем

$$
\int_{\xi_{k}}^{\xi_{k+1}}|g(x)| d x=\left|g\left(\theta_{k}\right)\right| \Delta \xi_{k}
$$

где $\theta_{k} \in\left(\xi_{k}, \xi_{k+1}\right), k \in \mathbb{Z}_{+}$. Отсюда, поскольку $\Delta \xi_{k} \gtrsim 1$, получаем

$$
S_{1}=\sum_{k=1}^{\infty}\left|g\left(\theta_{k}\right)\right| \lesssim \sum_{k=0}^{\infty}\left|g\left(\theta_{k}\right)\right| \Delta \xi_{k}=\sum_{k=0}^{\infty} \int_{\xi_{k}}^{\xi_{k+1}}|g(x)| d x=\int_{0}^{\infty}|g(x)| d x .
$$

Из равенства

$$
g\left(\xi_{k}\right)=g\left(\theta_{k}\right)-\int_{\xi_{k}}^{\theta_{k}} g^{\prime}(x) d x
$$


следует, что

$$
\begin{aligned}
& \left|g\left(\xi_{k}\right)\right| \leqslant\left|g\left(\theta_{k}\right)\right|+\int_{\xi_{k}}^{\theta_{k}}\left|g^{\prime}(x)\right| d x \leqslant\left|g\left(\theta_{k}\right)\right|+\int_{\xi_{k}}^{\xi_{k+1}}\left|g^{\prime}(x)\right| d x, \\
& S \leqslant \sum_{k=0}^{\infty}\left|g\left(\theta_{k}\right)\right|+\sum_{k=0}^{\infty} \int_{\xi_{k}}^{\xi_{k+1}}\left|g^{\prime}(x)\right| d x=S_{1}+\int_{0}^{\infty}\left|g^{\prime}(x)\right| d x .
\end{aligned}
$$

Таким образом,

$$
S \lesssim \int_{0}^{\infty}|g(x)| d x+\int_{0}^{\infty}\left|g^{\prime}(x)\right| d x=\frac{1}{2}\left(\|g\|_{L^{1}(\mathbb{R})}+\left\|g^{\prime}\right\|_{L^{1}(\mathbb{R})}\right) .
$$

Применяя неравенство Бернштейна (см. [7; гл. IV])

$$
\left\|g^{\prime}\right\|_{L^{1}(\mathbb{R})} \leqslant(d+2)\|g\|_{L^{1}(\mathbb{R})},
$$

получаем

$$
S \lesssim \frac{1+d+2}{2}\|g\|_{L^{1}(\mathbb{R})} \lesssim(1+d) \int_{\mathbb{R}_{+}}|f(x)| x^{\nu} d x .
$$

Учитывая, что $a_{k}=O\left(\xi_{k}^{\nu}\right)$ и $\xi_{k}^{\nu} \lesssim \omega\left(\xi_{k}\right), k \rightarrow \infty$, имеем

$$
\sum_{k=0}^{\infty} a_{k}\left|f\left(\xi_{k}\right)\right| \lesssim \sum_{k=0}^{\infty} \xi_{k}^{\nu}\left|f\left(\xi_{k}\right)\right| \lesssim \sum_{k=0}^{\infty}\left|g\left(\xi_{k}\right)\right|=S
$$

Тип $d$ в оценках появляется только после применения неравенства Бернштейна.

Лемма 3.5 доказана.

Отметим, что неравенство (3.10) можно записать в виде

$$
\|f\|_{1, d \alpha} \lesssim\|f\|_{1, x^{\nu}}
$$

где $\alpha(x)=\sum_{\xi_{k} \leqslant x} a_{k}$ - функция скачков. Неравенство (3.11) верно и в непрерывном случае $d \alpha(x)=a(x) d x, a(x)=O_{+\infty}\left(x^{\nu}\right)$.

Приведем еще один простой факт, вытекающий из леммы 3.5 :

$$
\lim _{\theta \rightarrow 1} \int_{\mathbb{R}_{+}} f(\theta x) d \alpha(x)=\int_{\mathbb{R}_{+}} f(x) d \alpha(x) .
$$

Действительно, для $\theta \in[1 / 2,3 / 2]$ имеем

$$
\begin{aligned}
I & =\left|\int_{\mathbb{R}_{+}} f(\theta x) d \alpha(x)-\int_{\mathbb{R}_{+}} f(x) d \alpha(x)\right| \leqslant\|f(\theta x)-f(x)\|_{1, d \alpha} \\
& \lesssim(1+\max \{\theta d, d\})\|f(\theta x)-f(x)\|_{1, x^{\nu}} .
\end{aligned}
$$

Отсюда для любого $\varepsilon$ больше нуля

$$
I \lesssim R^{\nu+1} \max _{0 \leqslant z \leqslant R}|f(\theta x)-f(x)|+\left(1+\frac{1}{\theta^{\nu+1}}\right) \int_{R}^{\infty}|f(x)| x^{\nu} d x<\varepsilon,
$$

если $R$ достаточно велико, а $\theta$ достаточно близко к 1 . 
Оценка (3.11) и равенство (3.12) справедливы для изучаемых нами мер $\sigma$.

Завершим доказательство теоремы 3.1. По лемме 3.4 она справедлива, если функция $f \in B_{2 \tau}^{0}$ удовлетворяет оценке (3.5).

Пусть теперь $f \in B_{2 \tau}^{0} \cap L_{x^{\nu}}^{1}\left(\mathbb{R}_{+}\right)$. Тогда в силу оценки $(3.11)$ и леммы 3.3 интеграл $\int_{\mathbb{R}_{+}} f d \sigma$ и ряд $\sum_{1}^{\infty} \gamma_{k} f\left(z_{k}\right)$ сходятся абсолютно.

Пусть $\theta \in[1 / 2,1), \varepsilon \in(0,1 / 2], \theta+\varepsilon \leqslant 1, a>\nu$ и $g(z)=f(\theta z) j_{a / 2}^{2}(\varepsilon \tau z)$. Тогда $g \in B_{2 \tau}^{0}, g=O_{+\infty}\left(x^{-a-1}\right)$ и можно применить лемму 3.4 :

$$
\int_{\mathbb{R}_{+}} f(\theta x) j_{a}^{2}(\varepsilon \tau x) d \sigma(x)=\sum_{k=1}^{\infty} \gamma_{k} f\left(\theta z_{k}\right) j_{a}^{2}\left(\varepsilon \tau z_{k}\right) .
$$

Осталось последовательно устремить $\varepsilon$ к 0 и $\theta$ к 1 , воспользовавшись при этом равенством (3.12).

Чтобы показать оптимальность типа $2 \tau$ в квадратурной формуле $(3.4)$, воспользуемся функцией

$$
f_{\varepsilon}(z)=\varphi^{2}(z) j_{a / 2}^{2}(\varepsilon z / 2) \in B_{2 \tau+\varepsilon}^{0}
$$

где $\varepsilon>0, a>0$. Имеем $f_{\varepsilon}=O_{+\infty}\left(x^{-\nu-a-1}\right), \sum_{1}^{\infty} \gamma_{k} f_{\varepsilon}\left(z_{k}\right)=0$, но $\int_{\mathbb{R}_{+}} f_{\varepsilon} d \sigma>0$.

Теорема 3.1 доказана.

ЗАмЕЧАНИЕ 3.2. Интерполяционные формулы по нулям целых функций хорошо изучены (см., например, $[23 ;$ гл. IV], [24; § 3.7]). Однако не каждая такая формула годится для построения оптимальной квадратурной формулы с положительными весами, поскольку должна выполняться лемма 3.2. А она существенно использует тот факт, что функция $\varphi$ является решением задачи Штурма-Лиувилля. Аналогичная ситуация наблюдается и в случае квадратурных формул на отрезке. Если система многочленов $\pi_{n}$ не является ортогональной и не удовлетворяет трехчленному рекуррентному соотношению, являющемуся аналогом задачи Штурма-Лиувилля, то по этой системе в общем случае нельзя построить квадратурную формулу наивысшей алгебраической степени точности с положительными весами.

\section{§4. Квадратурная формула Маркова}

Продолжаем использовать результаты предыдущих параграфов. Докажем квадратурную формулу Маркова вида (1.3) с фиксированным узлом в нуле. Для этого нам потребуются удовлетворяющие свойствам 1-5 собственные функции $\varphi^{0}(t, z)$ задачи Штурма-Лиувилля со спектральной функцией $d \sigma_{0}(x)=x^{2} d \sigma(x)$. Показатель $\nu_{0}$ для меры $\sigma_{0}$ в формуле (1.6) равен $\nu+2$.

Выведем задачу для $\varphi^{0}$ из задачи Штурма-Лиувилля (2.1), (2.2). Для этого положим

$$
v(t)=\frac{1}{W(t)} \int_{0}^{t} u(s) w(s) d s, \quad w_{0}(t)=\frac{W^{2}(t)}{w(t)}, \quad W(t)=\int_{0}^{t} w(s) d s, \quad t \in T .
$$


ЛЕмма 4.1. Функиия

$$
v=-z^{-2} w W^{-1} u^{\prime}
$$

является решением задачи Штурма-Лиувилля с дифференииальным уравнением

$$
\left(w_{0} v^{\prime}\right)^{\prime}+z^{2} w_{0} v=0, \quad t \in T,
$$

краевыми условиями вида (2.2), спектральной функцией $\sigma_{0}$ и собственными функицями

$$
\varphi^{0}(t, z)=\frac{1}{W(t)} \int_{0}^{t} \varphi(s, z) w(s) d s=-z^{-2} w(t) W^{-1}(t) \varphi_{t}^{\prime}(t, z),
$$

которые удовлетворяют свойствам 1-5.

ДокАзАТЕЛЬСтво. Интегрируя уравнение (2.1), получаем

$$
w u^{\prime}+z^{2} \int_{0}^{t} w u d s=0
$$

или $w u^{\prime}+z^{2} W v=0$ и $v=-z^{-2} w W^{-1} u^{\prime}$. При этом по определению $v$ имеем $u=w^{-1}(W v)^{\prime}$. Таким образом,

$$
\left(w^{-1}(W v)^{\prime}\right)^{\prime}+z^{2} w^{-1} W v=0 .
$$

Отсюда, раскрывая производные, находим

$$
\left(w^{-1}\right)^{\prime}\left(W^{\prime} v+W v^{\prime}\right)+w^{-1}\left(W^{\prime \prime} v+2 W^{\prime} v^{\prime}+W v^{\prime \prime}\right)+z^{2} w^{-1} W v=0
$$

или, с учетом равенства $W^{\prime}=w$,

$$
-w^{\prime} w^{-2}\left(w v+W v^{\prime}\right)+w^{-1}\left(w^{\prime} v+2 w v^{\prime}+W v^{\prime \prime}\right)+z^{2} w^{-1} W v=0 .
$$

После упрощений имеем

$$
\left(-w^{\prime} w^{-2} W+2\right) v^{\prime}+w^{-1} W v^{\prime \prime}+z^{2} w^{-1} W v=0 .
$$

Умножим это равенство на $W$ и учтем, что

$$
w_{0}^{\prime}=\left(w^{-1} W^{2}\right)^{\prime}=-w^{\prime} w^{-2} W^{2}+w^{-1} 2 W W^{\prime}=-w^{\prime} w^{-2} W^{2}+2 W .
$$

Получим $w_{0}^{\prime} v^{\prime}+w_{0} v^{\prime \prime}+z^{2} w_{0} v=0$ или $\left(w_{0} v^{\prime}\right)^{\prime}+z^{2} w_{0} v=0$.

Покажем, что $v(0)=u(0)$ и $v^{\prime}(0)=0$ (случай $t=l<+\infty$ рассматривается аналогично). Поскольку $u^{\prime}(0)=0$, в окрестности точки $t=0$ имеем $u(t)=$ $u(0)+o(t)$. Поэтому

$$
v(t)=W^{-1} \int_{0}^{t}(u(0)+o(s)) w d s=u(0)+o(t) .
$$

Теперь соотношение (4.1) для собственных функций очевидно. Найдем из него спектральную функцию. Для этого установим связь между преобразованиями Фурье задач Штурма-Лиувилля. При этом можно ограничиться плотными классами функций, удовлетворяющих теореме разложения. 
Пусть $f(t)$ - гладкая функция с компактным носителем. Тогда

$$
\widehat{f}_{w}(z)=\int_{T} f \varphi(t, z) w d t=\int_{T} f d\left(W \varphi^{0}\right)=\int_{T} g \varphi^{0}(t, z) w_{0} d t=\widehat{g}_{w_{0}}(z),
$$

где $g=-w W^{-1} f^{\prime}$. При этом

$$
f(t)=\int_{\mathbb{R}_{+}} \widehat{f}_{w} \varphi(t, z) d \sigma
$$

откуда в силу (4.1)

$$
g(t)=\int_{\mathbb{R}_{+}} \widehat{f}_{w}(z)\left(-w W^{-1}\right) \varphi_{t}^{\prime}(t, z) d \sigma=\int_{\mathbb{R}_{+}} \widehat{g}_{w_{0}}(z) \varphi^{0}(t, z) z^{2} d \sigma=\widetilde{h}_{d \sigma_{0}}(t),
$$

где $h=\widehat{g}_{w_{0}}$ и $d \sigma_{0}=z^{2} d \sigma$. Таким образом, свойства 4,5 можно считать выполненными.

Свойство 1 следует из общей теории задачи Штурма-Лиувилля. Имеем

$$
\varphi^{0}(t, z)=\prod_{k=1}^{\infty}\left(1-\frac{z^{2}}{\left(z_{k}^{0}\right)^{2}}\right) \in B_{t}^{0}
$$

где $z_{k}^{0}=z_{k}^{0}(t)$ - положительные нули по переменному $z$ производной $\varphi_{t}^{\prime}(t, z)$. Они являются собственными значениями задачи $\left(w_{0} v^{\prime}\right)^{\prime}+z^{2} w_{0} v=0$ на интервале $(0, t), v^{\prime}(0)=0, v(t)=0$. Так же, как и для нулей $t_{k}, z_{k}$, имеем $t_{k}^{0}\left(z_{k}^{0}\right)=t$, где $t_{k}^{0}(z)$ - нули уравнения $\varphi_{t}^{\prime}(t, z)=0$ и одновременно последовательные точки экстремума $\varphi(t, z)$. Поэтому нули $t_{k}, t_{k}^{0}$ и $z_{k}, z_{k}^{0}$ перемежаются: $t_{k}<t_{k}^{0}<t_{k+1}$, $z_{k}<z_{k}^{0}<z_{k+1}$.

Свойство 2 следует из равенства (4.1): для $t \in T, x \in \mathbb{R}_{+}$

$$
\left|\varphi^{0}(t, x)\right| \leqslant \frac{1}{W(t)} \int_{0}^{t}|\varphi(s, x)| w(s) d s \leqslant 1=\varphi^{0}(t, 0)=\varphi^{0}(0, x) .
$$

В дополнение к свойствам 1, 2 отметим связь представлений Мелера. Пусть справедливо представление (2.9). Тогда, используя определения $\varphi^{0}, W$ и переставляя пределы, находим

$$
\varphi^{0}(t, z)=\frac{1}{W(t)} \int_{0}^{t} w(s) \int_{0}^{s} \cos (z u) d \mu_{t}(u) d s=\int_{0}^{t} \cos (z u) d \mu_{t}^{0}(u),
$$

где

$$
d \mu_{t}^{0}(u)=\left(1-\frac{W(u)}{W(t)}\right) d \mu_{t}(u) \geqslant 0, \quad \int_{0}^{t} d \mu_{t}^{0}=1 .
$$

Для получения асимптотики $\varphi^{0}(t, z)$ (свойство 3 ) воспользуемся формулами (4.1) и (2.13) при $j=1$. Тогда соответственно имеем

$$
\begin{gathered}
z^{\nu_{0} / 2} \varphi^{0}(t, z)=-w(t) W^{-1}(t) z^{\nu / 2-1} \varphi_{t}^{\prime}(t, z), \\
z^{\nu_{0} / 2} \varphi^{0}(t, z)=C_{t}^{0}\left(\cos \left(t z-c_{t}^{0}\right)+O\left(|z|^{-1} e^{t|\operatorname{Im} z|}\right)\right),
\end{gathered}
$$


где $C_{t}^{0}=w W^{-1} C_{t}, c_{t}^{0}=c_{t}+\pi / 2$. Отметим, что если справедливо соотношение (2.14), то аналогично

$$
z^{-\nu_{0} / 2} C_{t}^{0} \sim \frac{(2 / \pi)^{1 / 2}}{\left(w_{0}(t) s_{0}(z)\right)^{1 / 2}} .
$$

Лемма 4.1 доказана.

Лемма 4.1 позволяет применить квадратурную формулу Гаусса с мерой $\sigma_{0}$ по нулям $z_{k}^{0}$. Из нее выведем квадратурную формулу Маркова.

Пусть $\tau \in T, \varphi^{0}(z)=\varphi^{0}(\tau, z)$ (имеем $\left.\varphi^{0}(x)=O_{+\infty}\left(x^{-\nu / 2-1}\right)\right), z_{k}^{0}=z_{k}^{0}(\tau)$,

$$
\gamma_{k}^{0}=\gamma_{k}^{0}(\tau)=\frac{2 z_{k}^{0}}{w_{0}(\tau)\left(\varphi^{0}\right)_{t}^{\prime}\left(\tau, z_{k}^{0}\right)\left(\varphi^{0}\right)_{z}^{\prime}\left(\tau, z_{k}^{0}\right)},
$$

и определим узлы $z_{k}^{\prime}=z_{k}^{\prime}(\tau)$ и веса $\gamma_{k}^{\prime}=\gamma_{k}^{\prime}(\tau)$ формулами

$$
\begin{gathered}
z_{0}^{\prime}=0, \quad \gamma_{0}^{\prime}=\int_{\mathbb{R}_{+}}\left(\varphi^{0}\right)^{2} d \sigma>0, \\
z_{k}^{\prime}=z_{k}^{0}, \quad \gamma_{k}^{\prime}=\left(z_{k}^{0}\right)^{-2} \gamma_{k}^{0}>0, \quad k \in \mathbb{N} .
\end{gathered}
$$

Теорема 4.1. Для $\tau \in T / 2$ и произвольной функции $f \in B_{2 \tau}^{0} \cap L_{x^{\nu}}^{1}\left(\mathbb{R}_{+}\right)$ справедлива квадратурная формула Маркова

$$
\int_{\mathbb{R}_{+}} f(x) d \sigma(x)=\sum_{k=0}^{\infty} \gamma_{k}^{\prime} f\left(z_{k}^{\prime}\right)
$$

где $\gamma_{0}^{\prime}=W^{-1}(\tau)$. Ряд в (4.2) сходится абсолютно.

Формула (4.2) оптимальна в том смысле, что для любого в больше нуля существует допустимая функиия $f_{\varepsilon} \in B_{2 \tau+\varepsilon}^{0} \cap L_{x^{\nu}}^{1}\left(\mathbb{R}_{+}\right)$, для которой равенство (4.2) неверно.

ДокАЗАТЕЛЬСтво. Пусть $f$ - допустимая функция и

$$
g(z)=z^{-2}\left\{f(z)-f(0)\left(\varphi^{0}(z)\right)^{2}\right\} \in B_{2 \tau}^{0} \cap L_{x^{\nu_{0}}}^{1}\left(\mathbb{R}_{+}\right) .
$$

Применим к функции $g$ формулу Гаусса с мерой $\sigma_{0}$

$$
\int_{\mathbb{R}_{+}} g(x) d \sigma_{0}(x)=\sum_{k=1}^{\infty} \gamma_{k}^{0} g\left(z_{k}^{0}\right)
$$

Имеем

$$
\int_{\mathbb{R}_{+}}\left\{f(x)-f(0)\left(\varphi^{0}(x)\right)^{2}\right\} d \sigma(x)=\sum_{k=1}^{\infty} \gamma_{k}^{0}\left(z_{k}^{0}\right)^{-2}\left\{f\left(z_{k}^{0}\right)-f(0)\left(\varphi^{0}\left(z_{k}^{0}\right)\right)^{2}\right\}
$$

или

$$
\int_{\mathbb{R}_{+}} f(x) d \sigma(x)=f(0) \int_{\mathbb{R}_{+}}\left(\varphi^{0}(x)\right)^{2} d \sigma(x)+\sum_{k=1}^{\infty} \gamma_{k}^{0}\left(z_{k}^{0}\right)^{-2} f\left(z_{k}^{0}\right) .
$$

Отсюда с учетом определения узлов $z_{k}^{\prime}$ и весов $\gamma_{k}^{\prime}$ получаем равенство (4.2). 
Покажем, что $\gamma_{0}^{\prime}=W^{-1}(\tau)$. Из формулы (4.1) следует, что

$$
\varphi^{0}(z)=\frac{1}{W(\tau)} \int_{0}^{\tau} \varphi(t, z) w(t) d t=\int_{T} h(t) \varphi(t, z) w(t) d t
$$

где $h(t)=W^{-1}(\tau) \chi_{[0, \tau]}(t)$. Таким образом, $\varphi^{0}=\widehat{h}$. Применяя равенство Парсеваля, получаем

$$
\gamma_{0}^{\prime}=\left\|\varphi^{0}\right\|_{2, d \sigma}^{2}=\|h\|_{2, w}^{2}=W^{-2}(\tau) \int_{0}^{\tau} w(t) d t=W^{-1}(\tau) .
$$

Для доказательства оптимальности типа $2 \tau$ для формулы (4.2) берем функцию вида $f_{\varepsilon}(z)=\left(\varphi^{0}(z)\right)^{2} j_{a / 2}^{2}(\varepsilon z / 2)$.

Теорема доказана.

\section{§ 5. Примеры}

Вначале сформулируем квадратурные формулы Бесселя в наших обозначениях. Они отвечают преобразованию Фурье-Ганкеля, связанному с многомерным анализом в $\mathbb{R}^{n}$. Затем приведем два интересных примера, также связанных с многомерным анализом на римановых многообразиях. Первый из них отвечает преобразованию Якоби и анализу на гиперболоидах $\mathbb{H}^{n}$. Второй пример связан с рядом Фурье по ортогональным многочленам Якоби и анализу на компактных римановых пространствах ранга 1, в частности, евклидовой сфере $\mathbb{S}^{n}$. В конце параграфа кратко рассмотрим общий случай задачи Штурма-Лиувилля с регулярным весом.

5.1. Преобразование Фурье-Ганкеля. Квадратурные формулы Бесселя, как отмечалось во введении, были построены в работах [8], [10], [11]. Приведем их в наших обозначениях в качестве следствия из теорем 3.1, 4.1. Для этого воспользуемся свойствами преобразования Фурье-Ганкеля и нормированной функции Бесселя, изложенными выше. В результате для $\alpha \geqslant-1 / 2$ имеем:

$$
\begin{gathered}
s_{\alpha}(x)=x^{2 \alpha+1}, \quad s_{\alpha 0}(x)=x^{2} s(x)=x^{2 \alpha+3}=s_{\alpha+1}(x), \quad x \in \mathbb{R}_{+}, \\
w_{\alpha}(t)=\frac{t^{2 \alpha+1}}{2^{2 \alpha} \Gamma^{2}(\alpha+1)}, \quad \varphi(t, z)=j_{\alpha}(t z), \quad t \in \mathbb{R}_{+}, \\
W(t)=\int_{0}^{t} w(s) d s=\frac{t^{2 \alpha+2}}{2^{2 \alpha} \Gamma^{2}(\alpha+1)(2 \alpha+2)}=\frac{t w(t)}{2 \alpha+2}, \\
w_{\alpha 0}(t)=\frac{W^{2}(t)}{w(t)}=\frac{t^{2} w(t)}{(2 \alpha+2)^{2}}=\frac{t^{2 \alpha+3}}{2^{2 \alpha+2} \Gamma^{2}(\alpha+2)}=w_{\alpha+1}(t), \\
\varphi^{0}(t, z)=-z^{-2} w(t) W^{-1}(t) \varphi_{t}^{\prime}(t, z)=-z^{-2} \frac{2 \alpha+2}{t}\left(j_{\alpha}(t z)\right)_{t}^{\prime}=j_{\alpha+1}(t z) .
\end{gathered}
$$

Отсюда следует, что задачи Штурма-Лиувилля для мер $d \sigma=s_{\alpha} d x$ и $d \sigma_{0}=$ $s_{\alpha+1} d x$ отличаются только индексами $\alpha$ и $\alpha+1$. Это значительно упрощает получение формул для узлов и весов формулы Маркова. 
Для $\tau>0, k \in \mathbb{N}$ имеем $\varphi(z)=j_{\alpha}(\tau z), \varphi_{k}(t)=j_{\alpha}\left(q_{k} t / \tau\right)$,

$$
\begin{gathered}
z_{k}=\frac{q_{k}}{\tau}, \quad \gamma_{k}=\frac{2 z_{k}}{w(\tau) \varphi_{k}^{\prime}(\tau) \varphi^{\prime}\left(z_{k}\right)}=\frac{2}{\tau^{2 \alpha+2}}\left(\frac{2^{\alpha+1} \Gamma(\alpha+2)}{q_{k} j_{\alpha+1}\left(q_{k}\right)}\right)^{2}, \\
z_{k}^{\prime}=z_{k}^{0}=\frac{q_{\alpha+1, k}}{\tau}, \quad \gamma_{k}^{\prime}=\left(z_{k}^{0}\right)^{-2} \gamma_{k}^{0}=\frac{\gamma_{\alpha+1, k}}{\left(z_{k}^{\prime}\right)^{2}} \\
z_{0}^{\prime}=0, \quad \gamma_{0}^{\prime}=W^{-1}(\tau)=\frac{2^{2 \alpha} \Gamma^{2}(\alpha+1)(2 \alpha+2)}{\tau^{2 \alpha+2}} .
\end{gathered}
$$

СлеДСтвИЕ 5.1. Для произвольной функиии $f \in B_{2 \tau}^{0} \cap L_{x^{2 \alpha+1}}^{1}\left(\mathbb{R}_{+}\right), \tau>0$, $\alpha \geqslant-1 / 2$, справедливы оптимальные по типу $2 \tau$ квадратурные формулы Гаусса и Маркова с положительными весами

$$
\int_{\mathbb{R}_{+}} f(x) x^{2 \alpha+1} d z=\sum_{k=1}^{\infty} \gamma_{k} f\left(z_{k}\right)=\sum_{k=0}^{\infty} \gamma_{k}^{\prime} f\left(z_{k}^{\prime}\right),
$$

где ряды сходятся абсолютно.

Это следствие полностью согласуется с результатами работ [8], [11] и теоремой 1.1, если учесть определение (1.11).

5.2. Преобразование Якоби. Необходимые свойства функций и преобразования Якоби возьмем из работ [25]-[29]. Приведем их практически дословно. При этом чуть подробнее остановимся на асимптотических свойствах функций Якоби. Затем вложим эти результаты в построенную выше схему.

Функции Якоби определяются при помощи гипергеометрической функции Гаусса равенством

$$
\varphi_{\lambda}^{(\alpha, \beta)}(t)=F\left(\frac{\rho+i \lambda}{2}, \frac{\rho-i \lambda}{2} ; \alpha+1 ;-(\operatorname{sh} t)^{2}\right),
$$

где все параметры комплексные, $\rho=\alpha+\beta+1$. Однако далее в примере предполагается, что $t \geqslant 0$ и $\alpha \geqslant \beta \geqslant-1 / 2$.

В частных случаях имеем

$$
\begin{gathered}
\varphi_{\lambda}^{(-1 / 2,-1 / 2)}(t)=\cos (\lambda t), \quad \varphi_{\lambda}^{(1 / 2,1 / 2)}(t)=\frac{2 \sin (\lambda t)}{\lambda \operatorname{sh} 2 t}, \\
\varphi_{\lambda}^{(\alpha, \alpha)}(t)=\varphi_{\lambda / 2}^{(\alpha,-1 / 2)}(2 t)=\frac{2^{\alpha} \Gamma(\alpha+1) P_{-1 / 2+i \lambda / 2}^{-\alpha}(\operatorname{ch} 2 t)}{(\operatorname{sh} 2 t)^{\alpha}},
\end{gathered}
$$

где $P_{\nu}^{\mu}-$ функция Лежандра.

Пусть $\varphi_{\lambda}(t)=\varphi_{\lambda}^{(\alpha, \beta)}(t)$. Прямое и обратное преобразования Якоби определяются соответственно равенствами

$$
J f(\lambda)=\int_{0}^{\infty} f(t) \varphi_{\lambda}(t) \Delta(t) d t, \quad J^{-1} f(t)=\int_{0}^{\infty} f(\lambda) \varphi_{\lambda}(t) s(\lambda) d \lambda,
$$

где

$$
\begin{gathered}
\Delta(t)=2^{2 \rho}(\operatorname{sh} t)^{2 \alpha+1}(\operatorname{ch} t)^{2 \beta+1}, \quad s(\lambda)=(2 \pi)^{-1}|c(\lambda)|^{-2}, \\
c(\lambda)=\frac{2^{\rho-i \lambda} \Gamma(\alpha+1) \Gamma(i \lambda)}{\Gamma((\rho+i \lambda) / 2) \Gamma((\rho+i \lambda) / 2-\beta)} .
\end{gathered}
$$


Преобразование Якоби является биективным преобразованием между пространством четных бесконечно дифференцируемых функций с компактным носителем и пространством четных быстро убывающих целых функций экспоненциального типа. Эта биекция расширяется до изоморфизма между пространствами $L_{\Delta}^{2}\left(\mathbb{R}_{+}\right)$и $L_{s}^{2}\left(\mathbb{R}_{+}\right)$. При этом справедливы равенства Парсеваля

$$
\|J f\|_{L_{s}^{2}\left(\mathbb{R}_{+}\right)}=\|f\|_{L_{\Delta}^{2}\left(\mathbb{R}_{+}\right)}, \quad\|f\|_{L_{s}^{2}\left(\mathbb{R}_{+}\right)}=\left\|J^{-1} f\right\|_{L_{\Delta}^{2}\left(\mathbb{R}_{+}\right)} .
$$

Теорема разложения является следствием этих утверждений.

Для $\alpha=\beta$ (случай функций Лежандра (5.3)) преобразование Якоби известно как преобразование Мелера-Фока (см. [30]). При $\alpha=\beta=-1 / 2$ в силу формул (5.2) и (5.4) преобразование Якоби сводится к косинус-преобразованию Фурье. Поэтому далее сосредоточимся на случае

$$
\alpha \geqslant \beta \geqslant-\frac{1}{2}, \quad \alpha>-\frac{1}{2} .
$$

Перечислим другие свойства функций Якоби.

1. Задача Штурма-Лиувилля: при $t, \lambda \geqslant 0$ функции $\varphi_{\lambda}(t)$ являются собственными функциями задачи

$$
\begin{gathered}
\frac{d}{d t}\left(\Delta(t) \frac{d}{d t} \varphi_{\lambda}(t)\right)+\left(\rho^{2}+\lambda^{2}\right) \Delta(t) \varphi_{\lambda}(t)=0, \\
\varphi_{\lambda}(0)=1, \quad \frac{d}{d t} \varphi_{\lambda}(0)=0 .
\end{gathered}
$$

2. Формула дифференцирования:

$$
\frac{d}{d t} \varphi_{\lambda}(t)=-\frac{\left(\rho^{2}+\lambda^{2}\right) \operatorname{sh} t \operatorname{ch} t}{2(\alpha+1)} \varphi_{\lambda}^{(\alpha+1, \beta+1)}(t) .
$$

3. Представление Мелера (см. [27]):

$$
\varphi_{\lambda}(t)=\frac{C_{\alpha}}{\Delta(t)} \int_{0}^{t} A_{\alpha, \beta}(s, t) \cos (\lambda s) d s, \quad A_{\alpha, \beta}(s, t) \geqslant 0,
$$

где константа $C_{\alpha}$ определена в формуле $(2.11)$ и

$$
\begin{aligned}
A_{\alpha, \beta}(s, t)=2^{\alpha+2 \beta+5 / 2} & \operatorname{sh}(2 t)(\operatorname{ch} t)^{\beta-\alpha}(\operatorname{ch}(2 t)-\operatorname{ch}(2 s))^{\alpha-1 / 2} \\
& \times F\left(\alpha+\beta, \alpha-\beta ; \alpha+\frac{1}{2} ; \frac{\operatorname{ch} t-\operatorname{ch} s}{2 \operatorname{ch} t}\right) .
\end{aligned}
$$

В частности, независимо, убеждаемся, что

$$
\varphi_{0}(t)=C_{\alpha}(\Delta(t))^{-1} \int_{0}^{t} A(s, t) d s>0
$$

при $t \geqslant 0$.

4. Асимптотика веса $s(\lambda)$. При $\lambda \rightarrow 0$

$$
|c(\lambda)|=c_{0} \lambda^{-1}(1+O(\lambda)), \quad c_{0}=\frac{2^{\rho} \Gamma(\alpha+1)}{\Gamma(\rho / 2) \Gamma(\rho / 2-\beta)} .
$$


Для $|z| \rightarrow \infty,|\arg z|<\pi$, имеем

$$
\ln \Gamma(z+a)=\left(z+a-\frac{1}{2}\right) \ln z-z+\frac{1}{2} \ln (2 \pi)+O\left(z^{-1}\right) .
$$

Отсюда следует, что при $|\lambda| \rightarrow \infty$

$$
\begin{gathered}
\ln c(\lambda)=(\rho+\alpha) \ln 2+\ln \Gamma(\alpha+1)-\left(\alpha+\frac{1}{2}\right) \ln (i \lambda)-\frac{1}{2} \ln (2 \pi)+O\left(|\lambda|^{-1}\right), \\
|c(\lambda)|=\frac{2^{\rho+\alpha} \Gamma(\alpha+1)(2 \pi)^{-1 / 2}}{\lambda^{\alpha+1 / 2}}\left(1+O\left(|\lambda|^{-1}\right)\right) .
\end{gathered}
$$

Таким образом, для $\lambda \in \mathbb{C},|\arg \lambda|<\pi$, имеем

$$
\begin{gathered}
s(\lambda)=(2 \pi)^{-1} c_{0}^{-2} \lambda^{2}(1+O(|\lambda|)), \quad|\lambda| \lesssim 1, \\
s(\lambda)=\left(2^{\rho+\alpha} \Gamma(\alpha+1)\right)^{-2} \lambda^{2 \alpha+1}\left(1+O\left(|\lambda|^{-1}\right)\right), \quad|\lambda| \gtrsim 1 .
\end{gathered}
$$

5. Асимптотики функций Якоби. В связи с приложениями многие авторы приводили асимптотические разложения функций Якоби (см., например, [31]). Мы затруднились найти их в нужной степени общности. Поэтому чуть подробнее остановимся на этом вопросе.

Имеем $F(a, b ; c ; z)=1+O(|z|),|z| \rightarrow 0$, и при $|z| \rightarrow \infty,|\arg (-z)|<\pi$,

$$
F(a, a ; c ; z)=\frac{\Gamma(c)}{\Gamma(a) \Gamma(c-a)}(-z)^{-a} \ln (-z)\left(1+O\left(|\ln (-z)|^{-1}\right)\right),
$$

если $c-a \notin \mathbb{Z}, a \neq 0$ (см. [32]). Положим здесь

$$
a=b=\frac{\rho}{2}, \quad c=\alpha+1, \quad z=-(\operatorname{sh} t)^{2} .
$$

Тогда

$$
\varphi_{0}(t)=1+O\left(t^{2}\right), \quad t \lesssim 1
$$

Далее при $t \rightarrow+\infty$

$$
\operatorname{sh} t=\operatorname{ch} t\left(1+O\left(e^{-2 t}\right)\right), \quad-z=(\operatorname{sh} t)^{2}=\frac{e^{2 t}}{4}\left(1+O\left(e^{-2 t}\right)\right) .
$$

Поэтому, учитывая определения (5.4), для $t \gtrsim 1$ имеем

$$
\varphi_{0}(t)=\frac{\Gamma(\alpha+1) 2 t}{\Gamma(\rho / 2) \Gamma(\rho / 2-\beta)(\operatorname{sh} t)^{\rho}}\left(1+O\left(t^{-1}\right)\right)=\frac{2 c_{0} t}{(\Delta(t))^{1 / 2}}\left(1+O\left(t^{-1}\right)\right) .
$$

В работе [33] доказано, что

$$
F\left(a+\mu, b-\mu ; c ; \frac{1-z}{2}\right)=\frac{\Gamma(c) 2^{(a+b-1) / 2}(\theta \operatorname{sh} \theta)^{1 / 2}\left(I_{c-1}(\gamma \theta)+\varepsilon_{1}\right)}{\gamma^{c-1}(z-1)^{c / 2}(z+1)^{(a+b+1-c) / 2}} .
$$

Здесь $z=\operatorname{ch} \theta, \gamma=(a-b) / 2+\mu$ и равномерно по $\theta$ и $\gamma$

$$
\left|\varepsilon_{1}\right| \leqslant \frac{A_{1} e^{A_{2} /|\gamma|}}{|\theta||\gamma|^{2}\left|K_{c}(\gamma \theta)\right|}, \quad|\arg (\gamma \theta)| \leqslant \frac{\pi}{2},
$$

где $A_{1}$ и $A_{2}$ - положительные константы, не зависящие от $\theta$ и $\gamma, I_{\nu}$ и $K_{\nu}-$ модифицированные функции Бесселя. 
Лемма 5.1. При $|\lambda| \rightarrow \infty, \operatorname{Re} \lambda \geqslant 0$, равномерно по $t>0$ имеем

$$
\begin{aligned}
\varphi_{\lambda}(t) & =\frac{1}{(\Delta(t) s(\lambda))^{1 / 2}}\left((\lambda t)^{1 / 2} J_{\alpha}(\lambda t)+O\left(|\lambda|^{-1} e^{t|\operatorname{Im} \lambda|}\right)\right) \\
& =\frac{(2 / \pi)^{1 / 2}}{(\Delta(t) s(\lambda))^{1 / 2}}\left(\cos \left(\lambda t-a_{\alpha}\right)+e^{t|\operatorname{Im} \lambda|} O\left(|\lambda t|^{-1}+|\lambda|^{-1}\right)\right),
\end{aligned}
$$

где константа $a_{\alpha}$ определена в формуле (1.15).

ДокАЗАТЕЛЬСтво. Положим в равенстве (5.12)

$$
a=b=\frac{\rho}{2}, \quad \mu=\frac{i \lambda}{2}, \quad c=\alpha+1, \quad \theta=2 t .
$$

Тогда

$$
\begin{gathered}
z=\operatorname{ch} 2 t, \quad \frac{1-z}{2}=-(\operatorname{sh} t)^{2}, \\
\gamma=\frac{i \lambda}{2}, \quad \gamma \theta=i \lambda t, \quad I_{c-1}(\gamma \theta)=i^{\alpha} J_{\alpha}(\lambda t) .
\end{gathered}
$$

Отсюда и из определения нормированной функции Бесселя (1.11) находим

$$
\begin{gathered}
\varphi_{\lambda}(t)=\frac{t^{\alpha+1 / 2}}{(\operatorname{sh} t)^{\alpha+1 / 2}(\operatorname{ch} t)^{\beta+1 / 2}}\left(j_{\alpha}(\lambda t)+\varepsilon\right), \\
\varepsilon=2^{\alpha} \Gamma(\alpha+1)(i \lambda t)^{-\alpha} \varepsilon_{1}, \quad|\varepsilon| \lesssim \frac{e^{2 A_{2} /|\lambda|}}{|\lambda|^{\alpha+2} t^{\alpha+1}\left|K_{\alpha+1}(i \lambda t)\right|},
\end{gathered}
$$

где $|\arg (i \lambda t)| \leqslant \pi / 2$, откуда $-\pi \leqslant \arg \lambda \leqslant 0$ или $\operatorname{Im} \lambda \leqslant 0$.

При $\alpha+1>-1 / 2$ и $|z| \rightarrow \infty,|\arg z|<3 \pi / 2$, имеем (см. [9])

$$
K_{\alpha+1}(z)=\sqrt{\frac{\pi}{2 z}} e^{-z}\left(1+O\left(|z|^{-1}\right)\right) .
$$

Следовательно, при $|\lambda| \rightarrow \infty, \operatorname{Re} \lambda \geqslant 0$ и $\operatorname{Im} \lambda \leqslant 0$ равномерно по $t>0$

$$
\left|K_{\alpha+1}(i \lambda t)\right| \asymp(|\lambda| t)^{-1 / 2} e^{-t|\operatorname{Im} \lambda|} .
$$

В результате для (5.14) имеем

$$
|\varepsilon| \lesssim \frac{e^{t|\operatorname{Im} \lambda|}}{|\lambda|(|\lambda| t)^{\alpha+1 / 2}}
$$

Теперь, применяя (1.11), (1.14), определения (5.4) и асимптотику веса $s(\lambda)$, приходим к асимптотикам (5.13). При этом в силу $\varphi_{\bar{\lambda}}(t)=\overline{\varphi_{\lambda}(t)}$ и аналогичного равенства для веса $s(\lambda)(5.4)$ условие $\operatorname{Im} \lambda \leqslant 0$ можно опустить.

Лемма 5.1 доказана.

Чтобы теперь выписать квадратурную формулу Якоби, необходимо преобразовать задачу Штурма-Лиувилля (5.6) в соответствии со схемой, изложенной 
в $\S 2$ на примере задачи с уравнением (2.3). Для этого введем модифицированные функции Якоби и вес

$$
\varphi(t, z)=\frac{\varphi_{z}(t)}{\varphi_{0}(t)}, \quad w(t)=\left(\varphi_{0}(t)\right)^{2} \Delta(t),
$$

где $t \in T=(0,+\infty), z=\lambda \in \mathbb{R}_{+}$. Вес $s=\sigma^{\prime}$ и спектр при этом не изменяются.

Из приведенных выше свойств функций Якоби имеем

$$
\begin{gathered}
w(t) \asymp \Delta(t) \asymp t^{2 \alpha+1}, \quad t \lesssim 1, \\
w(t) \asymp\left(t(\Delta(t))^{-1 / 2}\right)^{2} \Delta(t) \asymp t^{2}, \quad t \gtrsim 1,
\end{gathered}
$$

или $w(t) \asymp t^{(2 \alpha+1,2)}, t>0$, где $t^{(a, b)}$ обозначает кусочно-степенную функцию

$$
t^{(a, b)}= \begin{cases}t^{a}, & |t| \leqslant 1 \\ t^{b}, & |t| \geqslant 1\end{cases}
$$

Для веса $s$ имеем противоположное поведение $s(z) \asymp z^{(2,2 \alpha+1)}$. Таким образом, параметр $\nu$ как и в бесселевом случае для $\alpha>-1 / 2$ равен $\nu=2 \alpha+1>0$.

Отметим, что $w(t) s(1 / t) \asymp 1$ при $t>0$. Это свойство важно для приложений преобразования Якоби в гармоническом анализе, в частности, для доказательства неравенств Питта и их приложений (см. [34]-[36]).

Модифицированное преобразование Якоби определяется стандартными равенствами из $\S 2$. При этом имеем следующую простую связь между преобразованиями Якоби:

$$
\widehat{f}(z)=J\left[\varphi_{0}(t) f(t)\right](\lambda), \quad \tilde{f}(t)=\left(\varphi_{0}(t)\right)^{-1} J^{-1} f(t) .
$$

Отсюда следует необходимое равенство Парсеваля

$$
\|\widehat{f}\|_{L_{s}^{2}\left(\mathbb{R}_{+}\right)}=\|f\|_{L_{w}^{2}(T)}, \quad\|f\|_{L_{s}^{2}\left(\mathbb{R}_{+}\right)}=\|\widetilde{f}\|_{L_{w}^{2}(T)} .
$$

Выпишем еще основные свойства модифицированных функций Якоби при $\alpha \geqslant \beta \geqslant-1 / 2, \alpha>-1 / 2$. Используем свойства функций Якоби и результаты $\S 2$.

1. Функции $\varphi(t, z)$ являются собственными функциями задачи Штурма-Лиувилля

$$
\left(w u^{\prime}\right)^{\prime}+z^{2} u=0, \quad u(0)=1, \quad u^{\prime}(0)=0 .
$$

2. Справедливо представление Мелера

$$
\varphi(t, z)=\int_{0}^{t} a(s, t) \cos (z s) d s, \quad a(s, t)=\frac{A_{\alpha, \beta}(s, t)}{\int_{0}^{t} A_{\alpha, \beta}(u, t) d u} \geqslant 0 .
$$

Таким образом,

$$
\begin{gathered}
|\varphi(t, z)| \leqslant \varphi(t, 0)=\varphi(0, z)=1, \quad t \in T, \quad z \in \mathbb{R}_{+}, \\
\varphi(t, z)=\prod_{k=1}^{\infty}\left(1-\frac{z^{2}}{z_{k}^{2}(t)}\right)
\end{gathered}
$$

где $z_{k}(t)$ являются положительными нулями функции Якоби $\varphi_{z}(t)$. 
3. Из определения (5.15) по лемме 5.1 при $|z| \rightarrow \infty$, Re $z \geqslant 0$, и фиксированном $t>0$

$$
\varphi(t, z)=\frac{(2 / \pi)^{1 / 2}}{(w(t) s(z))^{1 / 2}}\left(\cos \left(t z-a_{\alpha}\right)+O\left(|z|^{-1} e^{t|\operatorname{Im} z|}\right)\right)
$$

где $s(z) \asymp z^{2 \alpha+1}=z^{\nu}$ при $|z| \rightarrow \infty$.

Асимптотические формулы для производных, как ранее отмечалось, вытекают из аналитичности собственных функций $\varphi$. Нужная асимптотика для $\varphi_{t}^{\prime}$ может быть получена также из (5.7) и (5.13).

Собственные функции связанной задачи Штурма-Лиувилля

$$
\varphi^{0}=-z^{-2} w W^{-1}(\varphi)_{t}^{\prime}=-z^{-2} w W^{-1}\left(\frac{\varphi_{z}}{\varphi_{0}}\right)_{t}^{\prime}=-z^{-2} \Delta W^{-1}\left(\left(\varphi_{z}\right)_{t}^{\prime} \varphi_{0}-\varphi_{z}\left(\varphi_{0}\right)_{t}^{\prime}\right),
$$

где $W(t)=\int_{0}^{t} w d s$, производные $\left(\varphi_{z}\right)_{t}^{\prime}$ в силу равенства (5.7) можно выразить через функции $\varphi_{z}^{(\alpha+1, \beta+1)}(t)$.

Таким образом, для модифицированного преобразования Якоби выполнены необходимые свойства и можно применить основные утверждения работы.

TEOPEMA 5.1. Пусть $\alpha \geqslant \beta \geqslant-1 / 2, \alpha>-1 / 2, u \varphi_{z}(t)=\varphi_{z}^{(\alpha, \beta)}(t)-\oint у н \kappa-$ иия Якоби. Тогда для произвольной функиии $f \in B_{2 \tau}^{0} \cap L_{x^{2 \alpha+1}}^{1}\left(\mathbb{R}_{+}\right), \tau>0$, справедливы оптимальные по типу $2 \tau$ квадратурные формулы Гаусса и Маркова с положительными весами

$$
\int_{\mathbb{R}_{+}} f(x) s_{\alpha, \beta}(x) d x=\sum_{k=1}^{\infty} \gamma_{k} f\left(z_{k}\right)=\sum_{k=0}^{\infty} \gamma_{k}^{\prime} f\left(z_{k}^{\prime}\right),
$$

где $z_{k} u z_{k}^{\prime}, k \in \mathbb{N},-$ положительные нули функиий $\varphi_{z}(\tau)$ u $\left(\varphi_{z} / \varphi_{0}\right)_{t}^{\prime}(\tau)$ cоответственно, $z_{0}^{\prime}=0, \gamma_{0}^{\prime}=W^{-1}(\tau)$. Ряды в них сходятся абсолютно.

Отметим, что преобразование Ганкеля и Якоби являются частными случаями преобразования Чебли-Тримеша (см. [37]), для которого многие необходимые свойства собственных функций задачи Штурма-Лиувилля известны. Интересно построить квадратурные формулы в этом случае.

5.3. Ряд Якоби. Приведем пример допустимой дискретной меры $\sigma$, связанной с рядом Фурье-Якоби (см. [9; гл. 10], [2]).

Пусть $\alpha \geqslant \beta \geqslant-1 / 2, T=(0, \pi)$. На интервале $T$ рассмотрим задачу Штурма-Лиувилля с дифференциальным уравнением Якоби

$$
\left(p v^{\prime}\right)^{\prime}+\left(z^{2}-\left(\frac{\rho}{2}\right)^{2}\right) p v=0, \quad v^{\prime}(0)=v^{\prime}(\pi)=0
$$

где $\rho=\alpha+\beta+1$,

$$
p(t)=2^{\rho} \sin ^{2 \alpha+1} \frac{t}{2} \cos ^{2 \beta+1} \frac{t}{2}, \quad t \in T .
$$

Как и в случае с преобразованием Якоби, удобно вначале привести свойства общепринятой задачи (5.18), а затем преобразовать ее, положив $u=v / v_{0}$ :

$$
\left(w u^{\prime}\right)^{\prime}+z^{2} w u=0, \quad u^{\prime}(0)=u^{\prime}(\pi)=0, \quad w=v_{0}^{2} p .
$$

При этом для удобства мы сразу обозначили общий спектр через $z$. 
Пусть $\pi_{k}(u), k \in \mathbb{Z}_{+}$, - ортогональные многочлены Якоби $P_{k}^{(\alpha, \beta)}(u)$ с весом $(1-u)^{\alpha}(1+u)^{\beta}, u \in[-1,1]$, и нормировкой $\pi_{k}(1)=1$. Тогда собственными функциями задачи (5.18), регулярными в краевых точках, являются косинус-многочлены $\pi_{k}(\cos t)$. При этом собственные значения находятся из равенства

$$
z^{2}-\left(\frac{\rho}{2}\right)^{2}=k(k+\rho) .
$$

Поэтому общей спектральной функцией $\sigma$ для задач (5.18) и (5.19) является функция скачков

$$
\sigma(z)=\sum_{\zeta_{k} \leqslant z} s_{k}, \quad \zeta_{k}=k+\frac{\rho}{2}, \quad k \in \mathbb{Z}_{+},
$$

где

$$
s_{k}=\left(\int_{0}^{\pi} \pi_{k}^{2}(\cos t) p(t) d t\right)^{-1}=2^{-\rho}(2 k+\alpha+\beta+1)\left(\begin{array}{c}
k+\alpha \\
k
\end{array}\right)\left(\begin{array}{c}
k+\alpha+\beta \\
k+\beta
\end{array}\right),
$$

кроме случая $\alpha=\beta=-1 / 2, k=0$, когда $s_{0}=1 / \pi$. Интеграл по мере $\sigma$ является взвешенной суммой по скачкам:

$$
\int_{\mathbb{R}_{+}} f(x) d \sigma(x)=\sum_{k=0}^{\infty} s_{k} f\left(\zeta_{k}\right) .
$$

Для $k \rightarrow \infty$ имеем

$$
\zeta_{k}=k+O\left(k^{-1}\right), \quad s_{k}=s\left(\zeta_{k}\right)\left(1+O\left(k^{-1}\right)\right) \asymp \zeta_{k}^{\nu},
$$

где $s(z)=2^{1-\rho} \Gamma^{-2}(\alpha+1) z^{2 \alpha+1}$ и $\nu=2 \alpha+1 \geqslant 0$.

Собственные функции задачи (5.18), удовлетворяющие начальным условиям $v(0)=1, v^{\prime}(0)=0$, выражаются через гипергеометрическую функцию:

$$
\phi_{z}(t)=\phi_{z}^{(\alpha, \beta)}(t)=F\left(\frac{\rho}{2}+z, \frac{\rho}{2}-z ; \alpha+1 ; \sin ^{2} \frac{t}{2}\right) .
$$

При этом $\pi_{k}(\cos t)=\phi_{\zeta_{k}}(t)$. Соответственно собственными функциями задачи (5.19) и весом будут

$$
\varphi(t, z)=\frac{\phi_{z}(t)}{\phi_{0}(t)}, \quad w=\phi_{0}^{2} p .
$$

Если $f \in L_{w}^{2}(T)$, то $\widehat{f}(z)=\int_{0}^{\pi} f(t) \varphi(t, z) w(t) d t$ и

$$
\widehat{f}\left(\zeta_{k}\right)=\int_{0}^{\pi} \phi_{0}(t) f(t) \pi_{k}(\cos t) p(t) d t=\widehat{g}_{k},
$$

где $\widehat{f}_{k}$ - коэффициенты Фурье-Якоби функции $g=\phi_{0} f$. При этом

$$
f(t)=\int_{\mathbb{R}_{+}} \widehat{f}(x) \varphi(t, x) d \sigma(x)
$$


или

$$
g(t)=\phi_{0}(t) \sum_{k=0}^{\infty} s_{k} \widehat{f}\left(\zeta_{k}\right) \varphi\left(t, \zeta_{k}\right)=\sum_{k=0}^{\infty} s_{k} \widehat{g}_{k} \pi_{k}(\cos t) .
$$

Это классический ряд Фурье-Якоби функции $g$, для которого теорема разложения общеизвестна.

Сравнивая определения собственных функций (5.20) и функций Якоби (5.1) для комплексных $t$ и $z$, имеем

$$
\begin{gathered}
\phi_{z}(t)=\varphi_{2 z / i}^{(\alpha, \beta)}\left(\frac{i t}{2}\right), \quad \Delta_{\alpha, \beta}\left(\frac{i t}{2}\right)=2^{\rho} i^{2 \alpha+1} p(t), \\
s_{\alpha, \beta}\left(\frac{2 z}{i}\right)=\frac{(2 z / i)^{2 \alpha+1}}{\left(2^{\rho+\alpha} \Gamma(\alpha+1)\right)^{2}}\left(1+O\left(|z|^{-1}\right)\right), \quad|z| \rightarrow \infty .
\end{gathered}
$$

Поэтому многие свойства этих функций похожи.

В частности, справедливо представление Мелера

$$
\phi_{z}(t)=\frac{C_{\alpha}}{p(t)} \int_{0}^{t} A(s, t) \cos (z s) d s,
$$

где

$$
\begin{gathered}
A(s, t)=2^{\beta+1 / 2} \sin t \cos ^{\beta-\alpha} \frac{t}{2}(\cos s-\cos t)^{\alpha-1 / 2} F\left(\alpha+\beta, \alpha-\beta ; \alpha+\frac{1}{2} ; \zeta\right), \\
\zeta=\frac{\cos (t / 2)-\cos (s / 2)}{2 \cos (t / 2)} .
\end{gathered}
$$

Здесь

$$
F\left(\alpha+\beta, \alpha-\beta ; \alpha+\frac{1}{2} ; \zeta\right)=\frac{F(\alpha+\beta, \beta+1 / 2 ; \alpha+1 / 2 ; \zeta /(\zeta-1))}{(1-\zeta)^{\alpha+\beta}}
$$

Для $\zeta \leqslant 0$ имеем $\zeta /(\zeta-1) \in[0,1)$, поэтому $A(s, t) \geqslant 0$.

Кроме того, в силу (5.12), как и при выводе (5.13), справедлива асимптотическая оценка

$$
\begin{gathered}
\phi_{z}(t)=\frac{t^{\alpha+1 / 2}}{2^{\alpha+1 / 2} \sin ^{\alpha+1 / 2}(t / 2) \cos ^{\beta+1 / 2}(t / 2)}\left(j_{\alpha}(t z)+\varepsilon\right), \\
|\varepsilon| \lesssim \frac{1}{t^{\alpha+1}|z|^{\alpha+2}\left|K_{\alpha+1}(-i t z)\right|}, \quad\left|K_{\alpha+1}(-i t z)\right| \asymp(t|z|)^{-1 / 2} e^{-t \operatorname{Im} z},
\end{gathered}
$$

где $|\arg (-i t z)| \leqslant \pi / 2$, откуда $0 \leqslant \arg \lambda \leqslant \pi$ или $\operatorname{Im} \lambda \geqslant 0$. Таким образом, при $|z| \rightarrow \infty, \operatorname{Re} z \geqslant 0$, фиксированном $t \in T$

$$
\phi_{z}(t)=\frac{(2 / \pi)^{1 / 2}}{(p(t) s(z))^{1 / 2}}\left(\cos \left(t z-a_{\alpha}\right)+O\left(|z|^{-1} e^{t|\operatorname{Im} z|}\right)\right),
$$

где $s(z)=2^{1-\rho} \Gamma^{-2}(\alpha+1) z^{\nu}$. Эта оценка согласуется с равенствами (5.21). 
Как и в случае преобразования Якоби для функций $\varphi(t, z)$ и $\varphi^{0}(t, z)$, необходимые свойства следуют из приведенных выше свойств функций $\phi_{z}(t)$. Отметим только, что

$$
W(t)=\int_{0}^{t} w d s=\int_{0}^{t} \phi_{0}^{2} p d s .
$$

Таким образом, для ряда Фурье-Якоби можно применить основные утверждения работы.

Теорема 5.2. Пусть $\alpha \geqslant \beta \geqslant-1 / 2 u \phi_{z}(t)=\phi_{z}^{(\alpha, \beta)}(t)-$ функция Якоби. Тогда для произвольной функиии $f \in B_{2 \tau}^{0} \cap L_{x^{2 \alpha+1}}^{1}\left(\mathbb{R}_{+}\right), \tau>0$, справедливы оптимальные по типу $2 \tau$ квадратурные формулы Гаусса и Маркова с положительными весами

$$
\sum_{k=0}^{\infty} s_{k} f\left(\zeta_{k}\right)=\sum_{k=1}^{\infty} \gamma_{k} f\left(z_{k}\right)=\sum_{k=0}^{\infty} \gamma_{k}^{\prime} f\left(z_{k}^{\prime}\right)
$$

где $z_{k}$ u $z_{k}^{\prime}, k \in \mathbb{N},-$ положительные нули функиий $\phi_{z}(\tau) u\left(\phi_{z} / \phi_{0}\right)_{t}^{\prime}(\tau)$ соответственно, $z_{0}^{\prime}=0, \gamma_{0}^{\prime}=W^{-1}(\tau)$. Все ряды сходятся абсолютно.

В качестве тестового примера рассмотрим случай $\alpha=\beta=-1 / 2$. Имеем косинус-задачу Штурма-Лиувилля

$$
\begin{aligned}
& u^{\prime \prime}+z^{2} u=0, \quad u^{\prime}(0)=u^{\prime}(\pi)=0, \quad w(t)=1, \\
& \varphi(t, z)=\cos (t z), \quad \zeta_{k}=k, \quad \pi_{k}(\cos t)=\cos (k t), \\
& s_{k}=\|\cos (k t)\|_{L^{2}(0, \pi)}^{-2}=\left\{\begin{array}{ll}
\frac{1}{\pi}, & k=0, \\
\frac{2}{\pi}, & k>0,
\end{array} \quad \nu=0 .\right.
\end{aligned}
$$

Для связанной задачи Штурма-Лиувилля имеем $W=\int_{0}^{t} d s=t, w_{0}=t^{2}$ и

$$
\varphi^{0}(t, z)=\frac{1}{t} \int_{0}^{t} \cos (z s) d s=\frac{\sin (z t)}{z t}=-z^{2} t^{-1}(\cos (z t))_{t}^{\prime} .
$$

Пусть $\tau \in T=(0, \pi)$. Имеем

$$
\begin{gathered}
\varphi_{t}^{\prime}(t, z)=-z \sin (t z), \quad \varphi_{z}^{\prime}(t, z)=-t \sin (t z), \\
z_{k}(\tau)=\frac{\pi(k-1 / 2)}{\tau}, \quad \varphi_{k}^{\prime}(\tau)=-z_{k} \sin \left(\tau z_{k}\right), \quad \varphi^{\prime}\left(z_{k}\right)=-\tau \sin \left(\tau z_{k}\right),
\end{gathered}
$$

поэтому

$$
\gamma_{k}(\tau)=\frac{2 z_{k}}{w(\tau) \varphi_{k}^{\prime}(\tau) \varphi^{\prime}\left(z_{k}\right)}=\frac{2}{\tau} .
$$

Аналогично для $k \in \mathbb{N}$

$$
z_{k}^{\prime}=z_{k}^{0}=\frac{\pi k}{\tau}, \quad \gamma_{k}^{0}=\frac{2 z_{k}^{0}}{w_{0}(\tau)\left(\varphi^{0}\right)_{t}^{\prime}\left(\tau, z_{k}^{0}\right)\left(\varphi^{0}\right)_{z}^{\prime}\left(\tau, z_{k}^{0}\right)}=\frac{2(\pi k)^{2}}{\tau^{3}}, \quad \gamma_{k}^{\prime}=\frac{2}{\tau} .
$$

При $k=0$ имеем $z_{0}^{\prime}=0, \gamma_{0}^{\prime}=W^{-1}(\tau)=\tau^{-1}$. 
Пусть $f \in B_{2 \tau}^{0} \cap L_{d \sigma}^{1}\left(\mathbb{R}_{+}\right), \tau \in T / 2=(0, \pi / 2)$. В данном случае это означает, что

$$
f \in B_{2 \tau}^{0}, \quad|f(0)|+2 \sum_{k=1}^{\infty}|f(k)|<\infty .
$$

Тогда справедливы квадратурные формулы Гаусса и Маркова

$$
\frac{1}{\pi} f(0)+\frac{2}{\pi} \sum_{k=1}^{\infty} f(k)=\frac{2}{\tau} \sum_{k=1}^{\infty} f\left(\frac{\pi(k-1 / 2)}{\tau}\right)=\frac{1}{\tau} f(0)+\frac{2}{\tau} \sum_{k=1}^{\infty} f\left(\frac{\pi k}{\tau}\right) .
$$

Отметим, что эти равенства являются простым следствием формулы суммирования Пуассона (1.9): все они равны

$$
\pi^{-1} \widehat{f}(0)=2 \pi^{-1} \int_{\mathbb{R}_{+}} f d z .
$$

5.4. Задача Штурма-Лиувилля с регулярным весом. Выше были рассмотрены сингулярные задачи Штурма-Лиувилля, когда вес $w$ дифференциального уравнения обращается в нуль на границе интервала $T$. Больше общей информации представлено для случая регулярного веса $w$, когда $w>0$ на границе T (см. [12], [19], [20], [38], [39]).

Для простоты рассмотрим случай $T=(0,+\infty)$ и непрерывного спектра. В приведенных источниках задача Штурма-Лиувилля изучается в виде

$$
y^{\prime \prime}(t)+(\lambda-q(t)) y(t)=0
$$

с краевым условием

$$
y^{\prime}(0)-h y(0)=0, \quad 0 \leqslant h<+\infty,
$$

или

$$
y(0)=0, \quad h=+\infty .
$$

Отметим, что случай $h=+\infty$ исследован не так подробно.

Пусть спектр задачи $\lambda=z^{2} \geqslant 0$, спектральная функция $\sigma \in C^{1}\left(\mathbb{R}_{+}\right)$и вес $s=\sigma^{\prime}$. Достаточные условия на функцию $q$, когда выполняются эти предположения, можно найти в приведенных источниках.

Через $y(t, z)$ обозначим решение задачи Штурма-Лиувилля с начальными условиями $y(0, z)=1, y^{\prime}(0, z)=h$ для $h<+\infty$ или $y(0, z)=0, y^{\prime}(0, z)=1$ для $h=+\infty$ соответственно. Тогда известно из [12], [38], что при $|z| \rightarrow \infty$

$$
y(t, z)=\cos (t z)+O\left(|z|^{-1} e^{t|\operatorname{Im} z|}\right), \quad s(z)=\frac{2}{\pi}\left(1+O\left(|z|^{-1}\right)\right)
$$

для $h<+\infty$ и

$$
z y(t, z)=\cos \left(t z-\frac{\pi}{2}\right)+O\left(|z|^{-1} e^{t|\operatorname{Im} z|}\right), \quad s(z)=\frac{2}{\pi} z^{2}\left(1+O\left(|z|^{-1}\right)\right)
$$

для $h=+\infty$. При этом для $h<+\infty$

$$
y(t, z)=\cos (t z)+\int_{0}^{t} L_{1}(t, u) \cos (z u) d u,
$$

где ядро $L_{1} \geqslant 0$, если, например, $q \geqslant 0$ (см. [39]). 
Свойства нулей собственных функций и достаточные условия справедливости теоремы разложения также общеизвестны.

Для сведения задачи к стандартной вновь полагаем $u=y / y_{0}$. В случае $h<+\infty$ удобно взять $y_{0}(t)=y(t, 0)$, тогда собственными функциями стандартной задачи будут $\varphi(t, z)=y(t, z) / y(t, 0)$. Спектральная функция и спектр не изменяются, вес $w=y_{0}^{2}$,

$$
\begin{gathered}
\varphi(t, z)=\frac{(2 / \pi)^{1 / 2}}{(w(t) s(z))^{1 / 2}}\left(\cos (t z)+O\left(|z|^{-1} e^{t|\operatorname{Im} z|}\right)\right), \\
\varphi(t, z)=\int_{0}^{t} \cos (z u) d \mu_{t}(u) .
\end{gathered}
$$

Связанная задача Штурма-Лиувилля вводится обычным образом.

В качестве примера, который можно вложить в нашу схему, упомянем преобразование Вебера-Орра (см. [20; §21], [9; гл. 7]):

$$
f(t)=\int_{0}^{\infty} \widehat{f}(x) y(t, x) x d x, \quad \widehat{f}(x)=\int_{a}^{\infty} f(t) y(t, x) d t
$$

где

$$
y(t, z)=\frac{\sqrt{t}\left(J_{\alpha}(t z) Y_{\alpha}(a z)-J_{\alpha}(a z) Y_{\alpha}(t z)\right)}{\left\{\left(J_{\alpha}(a z)\right)^{2}+\left(Y_{\alpha}(a z)\right)^{2}\right\}^{1 / 2}}, \quad a>0 .
$$

В этом случае имеем дифференциальное уравнение Бесселя

$$
y^{\prime \prime}+\left(z^{2}-\frac{\alpha^{2}-1 / 4}{t^{2}}\right) y=0, \quad t \geqslant a, \quad y(a)=0 .
$$

Для сведения задачи к стандартной требуется сдвинуть аргумент $t$ и выполнить описанные преобразования.

\section{Список литературы}

[1] В.И. Крылов, Приближенное вычисление интегралов, Физматгиз, М., 1959, 327 с.; англ. пер.: V. I. Krylov, Approximate calculation of integrals, The Macmillan Co., New York-London, 1962, $\mathrm{x}+357$ pp.

[2] Г. Сеге, Ортогональные многочлены, Физматгиз, М., 1962, 500 с.; пер. с англ.: G. Szegö, Orthogonal polynomials, rev. ed., Amer. Math. Soc. Colloq. Publ., 23, Amer. Math. Soc., Providence, R.I., 1959, ix+421 pp.

[3] W. Gautschi, "Orthogonal polynomials and quadrature", Electron. Trans. Numer. Anal., 9 (1999), 65-76 (electronic).

[4] Д. В. Горбачев, В. И. Иванов, "Одна экстремальная задача для многочленов, связанная с кодами и дизайнами”, Матем. заметки, 67:4 (2000), 508-513; англ. пер.: D. V. Gorbachev, V.I. Ivanov, "An extremum problem for polynomials related to codes and designs", Math. Notes, 67:4 (2000), 433-438.

[5] В.А. Юдин, "Код и дизайн", Дискрет. матем., 9:2 (1997), 3-11; англ. пер.: V. A. Yudin, "Codes and designs", Discrete Math. Appl., 7:2 (1997), 147-155.

[6] V. I. Levenshtein, "Universal bounds for codes and designs", Handbook of coding theory, v. 1, North-Holland, Amsterdam, 1998, 499-648.

[7] Н. И. Ахиезер, Лекиии по теории аппроксимации, 2-е изд., Наука, М., 1965, 407 с.; нем. пер.: N. I. Achieser, Vorlesungen über Approximationstheorie, Math. Lehrbücher und Monogr., II, Akademie-Verlag, Berlin, 1967, xiii+412 pp. 
[8] C. Frappier, P. Oliver, "A quadrature formula involving zeros of Bessel functions", Math. Comp., 60:201 (1993), 303-316.

[9] Г. Бэйтмен, А. Эрдейи, Высшие трансиендентные функиии, т. 2, Наука, М., 1966, 295 с.; пер. с англ.: A. Erdélyi, W. Magnus, F. Oberhettinger, F. G. Tricomi, Higher transcendental functions, v. II, Based, in part, on notes left by H. Bateman, McGraw-Hill Book Company, Inc., New York-Toronto-London, 1953, xvii+396 pp.

[10] G. R. Grozev, Q. I. Rahman, "A quadrature formula with zeros of Bessel functions as nodes", Math. Comp., 64:210 (1995), 715-725.

[11] R. B. Ghanem, C. Frappier, "Explicit quadrature formulae for entire functions of exponential type", J. Approx. Theory, 92:2 (1998), 267-279.

[12] Б. М. Левитан, И. С. Саргсян, Введение в спектральную теорию, Наука, М., 1970, 672 с.; англ. пер.: В. M. Levitan, I. S. Sargsjan, Introduction to spectral theory: selfadjoint ordinary differential operators, Transl. Math. Monogr., 39, Amer. Math. Soc., Providence, R.I., 1975, xi+525 pp.

[13] Д. В. Горбачев, “Экстремальная задача для целых функций экспоненциального сферического типа, связанная с оценкой Левенштейна плотности упаковки $\mathbb{R}^{n}$ шарами", Изв. ТулГУ. Сер. Матем. Мех. Информ., 6:1 (2000), 71-78.

[14] Д. В. Горбачев, "Экстремальные задачи для целых функций экспоненциального сферического типа", Матем. заметки, 68:2 (2000), 179-187; англ. пер.: D. V. Gorbachev, "Extremum problems for entire functions of exponential spherical type", Math. Notes, 68:2 (2000), 159-166.

[15] Д. В. Горбачев, "Экстремальная задача для периодических функций с носителем в шаре", Матем. заметки, 69:3 (2001), 346-352; англ. пер.: D. V. Gorbachev, "Extremum problem for periodic functions supported in a ball", Math. Notes, 69:3 (2001), 313-319.

[16] А. В. Иванов, В.И. Иванов, "Оптимальные аргументы в неравенстве Джексона в пространстве $L_{2}\left(\mathbb{R}^{d}\right)$ со степенным весом", Матем. заметки, 94:3 (2013), 338-348; англ. пер.: А. V. Ivanov, V. I. Ivanov, "Optimal arguments in Jackson's inequality in the power-weighted space $L_{2}\left(\mathbb{R}^{d}\right)$ ", Math. Notes, 94:3 (2013), 320-329.

[17] Р. Курант, Д. Гильберт, Методы математической физики, т. 1, ГТТИ, М.-Л., 1933, 528 с.; пер. с нем.: R. Courant, D. Hilbert, Methoden der mathematischen Physik, v. I, Grundlehren Math. Wiss., 12, J. Springer, Berlin, 1931, xiv+469 pp.

[18] Э. Ч. Титчмарш, Разложения по собственным функциям, связанные с дифференииальными уравнениями второго порядка, т. 1, ИЛ, М., 1960, 278 с.; пер. с англ.: E. C. Titchmarsh, Eigenfunction expansions associated with second-order differential equations, Clarendon Press, Oxford, 1946, 175 pp.

[19] Б. М. Левитан, И. С. Саргсян, Операторы Штурма-Лиувилля и Дирака, Наука, M., 1988, 432 с.; англ. пер.: B. M. Levitan, I. S. Sargsjan, Sturm-Liouville and Dirac operators, Math. Appl. (Soviet Ser.), 59, Kluwer Academic Publishers Group, Dordrecht, 1991, xii+350 pp.

[20] М.А. Наймарк, Линейные дифференциальные операторы, 2-е изд., Наука, М., 1969, 526 с.; англ. пер. 1-го изд.: М. A. Naimark, Linear differential operators, v. I, II, Frederick Ungar Publishing Co., New York, 1967, 1968, xiii+144 pp., xv+352 pp.

[21] Ф. Олвер, Введение в асимптотические методы и специальне функиии, Наука, M., 1978, 375 с.; пер. с англ.: F. W. J. Olver, Introduction to asymptotics and special functions, Academic Press, New York, 1974, xii+297 pp.

[22] С. М. Никольский, Приближение функиий многих переменных и теоремы вложения, 2-е изд., Наука, М., 1977, 455 с.; англ. пер. 1-го изд.: S. M. Nikol'skii, Approximation of functions of several variables and imbedding theorems, Grundlehren Math. Wiss., 205, Springer-Verlag, New York-Heidelberg, 1975, viii+418 pp. 
[23] Б. Я. Левин, Распределение корней целых функиий, Гостехиздат, М., 1956, 632 с.; англ. пер.: В. Ja. Levin, Distribution of zeros of entire functions, Amer. Math. Soc., Providence, R.I., 1964, viii+493 pp.

[24] М. А. Евграфов, Асимптотические оценки и целье функиии, 3-е изд., Наука, М., 1979, 320 с.; англ. пер. 1-го изд.: М. A. Evgrafov, Asymptotic estimates and entire functions, Gordon and Breach, Inc., New York, 1961, x+181 pp.

[25] M. Flensted-Jensen, T. H. Koornwinder, "The convolution structure for Jacobi function expansions", Ark. Mat., 11:1-2 (1973), 245-262.

[26] M. Flensted-Jensen, T. H. Koornwinder, "Jacobi functions: the addition formula and the positivity of the dual convolution structure", Ark. Mat., 17:1-2 (1979), 139-151.

[27] T. Koornwinder, "A new proof of a Paley-Wiener type theorem for the Jacobi transform", Ark. Mat., 13:1-2 (1975), 145-159.

[28] T.H. Koornwinder, "Jacobi functions and analysis on noncompact semisimple Lie groups", Special functions: group theoretical aspects and applications, Math. Appl., Reidel, Dordrecht, 1984, 1-85.

[29] N. Ben Salem, K. Trimèche, "Mehler integral transforms associated with Jacobi functions with respect to the dual variable", J. Math. Anal. Appl., 214:2 (1997), 691-720.

[30] Н. Я. Виленкин, Специальные функиии и теория представления групп, 2-е изд., Наука, М., 1991, 576 с.; англ. пер. 1-го изд.: N. Ya. Vilenkin, Special functions and the theory of group representations, Transl. Math. Monogr., 22, Amer. Math. Soc., Providence, R.I., 1968, x+613 pp.

[31] W. O. Bray, M. A. Pinsky, "Pointwise Fourier inversion on rank one symmetric spaces and related topics", J. Funct. Anal., 151:2 (1997), 306-333.

[32] Г. Бэйтмен, А. Эрдейи, Высшие трансцендентные функиии, т. 1, Наука, М., 1965, 294 с.; пер. с англ.: A. Erdélyi, W. Magnus, F. Oberhettinger, F. G. Tricomi, Higher transcendental functions, v. I, Based, in part, on notes left by H. Bateman, McGraw-Hill Book Company, Inc., New York-Toronto-London, 1953, xxvi+302 pp.

[33] D. S. Jones, "Asymptotics of the hypergeometric function", Math. Methods Appl. Sci., 24:6 (2001), 369-389.

[34] L. De Carli, D. Gorbachev, S. Tikhonov, "Pitt and Boas inequalities for Fourier and Hankel transforms", J. Math. Anal. Appl., 408:2 (2013), 762-774.

[35] D. Gorbachev, E. Liflyand, S. Tikhonov, "Weighted Fourier inequalities: Boas' conjecture in $\mathbb{R}^{n}$, J. Anal. Math., 114:1 (2011), 99-120.

[36] D. Gorbachev, S. Tikhonov, "Moduli of smoothness and growth properties of Fourier transforms: two-sided estimates", J. Approx. Theory, 164:9 (2012), 1283-1312.

[37] N.B. Andersen, "On real Paley-Wiener theorems for certain integral transforms", J. Math. Anal. Appl., 288:1 (2003), 124-135.

[38] Б. М. Левитан, “Об асимптотическом поведении спектральной функции и о разложении по собственным функциям самосопряженного дифференциального уравнения второго порядка. II", Изв. АН СССР. Сер. матем., 19:1 (1955), 33-58.

[39] Б. М. Левитан, Теория операторов обобщенного сдвига, Наука, М., 1973, 312 с.

\section{Дмитрий Викторович Горбачев (Dmitriy V. Gorbachev)}

Тульский государственный университет

E-mail: dvgmail@mail.ru

\section{Валерий Иванович Иванов \\ (Valeriy I. Ivanov)}

Тульский государственный университет

E-mail: ivaleryi@mail.ru
Поступила в редакцию 31.07.2014 и 14.11.2014 